\title{
ANCIENT SOLUTIONS ON BUNDLES WITH NON-ABELIAN STRUCTURAL GROUP
}

\author{
PENG LU AND Y. K. WANG
}

\begin{abstract}
We generalize the ancient solutions of the Ricci flow on certain principal SO(3) bundles over compact quaternionic Kähler manifolds constructed by Bakas, Kong, and $\mathrm{Ni}$ to certain $\mathbb{R P}^{3}$ fibre bundles over a product of two compact quaternionic Kähler manifolds. The ancient solutions are of Type I, $\kappa$-noncollapsed, and have positive Ricci curvature. Analogous solutions on even-dimensional nonKählerian bundle spaces are also constructed.
\end{abstract}

Keywords. Ricci flow, ancient solutions, $\mathbb{R P}^{3}$ fibre bundles, quaternionic Kähler manifolds, Riemannian submersions

MSC (2010) 53C44

\section{Introduction}

Ancient solutions for the Ricci flow arise naturally when one blows up the finite time singularities of Ricci flow solutions on closed manifolds. The work in BKN12 and DHS12 shows that ancient solutions are interesting objects in geometric analysis.

In [LW16] we generalized the examples in [BKN12, §5] of ancient solutions on principal circle bundles over Fano Kähler-Einstein manifolds to the situation of principal torus bundles over a finite product of Fano Kähler-Einstein manifolds. In this paper we shall generalize in a similar fashion the ancient solutions given by Theorem 6.2 in BKN12 on certain principal $\mathrm{SO}(3)$ bundles over compact quaternionic Kähler manifolds (abbreviated as QK manifolds in the sequel).

To describe our results, let us choose QK manifolds $\left(M_{i}^{4 n_{i}}, g_{i}\right), i=1, \cdots, m$, with $m \geq 2$. Recall that for each such space there is an associated principal $\mathrm{SO}(3)$ bundle $P_{i}$ over $M_{i}$ whose associated $\mathbb{R}^{3}$ bundle is the analog of the anti-canonical bundle for the Kähler-Einstein case. Taking the product of these principal bundles we can form an associated $\frac{\mathrm{SO}(3) \times \cdots \times \mathrm{SO}(3)}{\Delta \mathrm{SO}(3)}$ fibre bundle $\bar{P}$ over the product $M_{1} \times \cdots \times M_{m}$ (see $\$ 2.1$ for further details). By [Wa92], there exist connection type Einstein metrics on $\bar{P}$. As in [BKN12], we exploit the fact that the Ricci flow preserves the family of connection type metrics, and so the search for ancient solutions of connection type on $\bar{P}$ is reduced to the search for special flow lines for a reduced system of ordinary differential equations (ODEs in short).

In this paper we will construct continuous families of $\kappa$-noncollapsed type I ancient solutions when $m=2$ and for a special class of bundles when $m \geq 3$. Below we give a description of the $m=2$ case, and refer the reader to Theorem [5.6 for our results

Date: revised May 27, 2018.

P.L. is partially supported by Simons Foundation through Collaboration Grant 229727. Y.K.W. is partially supported by NSERC Grant No. OPG0009421. 
in the $m \geq 3$ case. Note that $\frac{\mathrm{SO}(3) \times \mathrm{SO}(3)}{\Delta \mathrm{SO}(3)}$ is diffeomorphic to $\mathbb{R P}^{3}$, and so the ancient solutions exist on $\mathbb{R P}^{3}$ fibre bundles.

Main Theorem. There are ( $i$ ) a continuous 1-parameter family of ancient solutions and (ii) two distinct ancient solutions of the Ricci flow on the $\mathbb{R P}^{3}$ fibre bundle $\bar{P}$ over an arbitrary product $M_{1} \times M_{2}$ of $Q K$ manifolds with positive scalar curvature. These solutions are of type I when time $t \rightarrow-\infty$ and are $\kappa$-noncollapsed at all scales for some $\kappa>0$. Their rescaled backwards limits are Einstein metrics. Furthermore, the solutions in both $(i)$ and (ii) have positive Ricci curvature for $t$ close to $-\infty$.

Technically, the difference between our work and that in BKN12 is that the ODE system in [BKN12] is two-dimensional, has a first integral, and hence is integrable. For our system, there is no longer an obvious first integral, and the two Einstein metrics on the bundle $\bar{P}$ are only established implicitly. To get ancient solutions whose rescaled backwards limits are these Einstein metrics, by the Hartman-Grobman Theorem, it suffices for us to show the existence of unstable directions of the linearization of our system about these Einstein metrics. Thus one needs to achieve this using appropriate estimates for their location since they are not given explicitly. Furthermore, for some of these ancient solutions, we will consider their singularity formulation in finite time, which requires us to find suitable bounded sets and differential inequalities and use them to show that the solutions stay in these sets. The geometric properties of the solutions are then established from various estimates derived from the ODEs.

Compared to the work in [LW16], the analysis here is more delicate because the bundle $\bar{P}$ admits two Einstein metrics. One of the Einstein metrics takes the place of the origin in the system considered in [LW16], which is a source for the Ricci flow. The origin is then an additional stationary solution for the system in this paper. The fact that the origin is actually a sink for the Ricci flow means that we do not get collapsed solutions anymore. This can perhaps be regarded as an indication through the Ricci flow that bundles with non-abelian structural groups are more rigid than those with abelian structural groups.

The ancient solutions in $\$ 5$ include continuous families which exist on even dimensional non-Kählerian manifolds. The only examples of this type in the literature that we are aware of are a 2-parameter family of left-invariant solutions found in La13. on each of the compact connected simple Lie groups except $\operatorname{Sp}(n)$.

As in [LW16], we also obtain without additional work the existence of pseudoRiemannian ancient and immortal solutions if we replace some or all of the QK factors of positive scalar curvature by ones with negative scalar curvature (see Remark 3.2).

The following is an outline of the rest of this paper. In $\$ 2$ we derive the ODE for the Ricci flow and establish properties about the linearization of the ODE at Einstein metrics (Proposition [2.6). In \$3 we prove the existence of ancient solutions on the $\mathbb{R P}^{3}$ fibre bundles over a product of two QK manifolds (Theorem 3.1), and discuss their metric behavior as time $t \rightarrow-\infty$ and their $\kappa$-noncollapsed property (Theorem 3.5 and 3.8, and Corollary 3.9). In $\$ 4$ we prove a general result (Theorem 4.2) which enables us to use the metric tensor limits of the previous section to conclude (without any computations) that the corresponding ancient solutions are of Type I as time approaches $-\infty$. We also show that the ancient solutions for the $m=2$ case have positive Ricci curvature when time is close to $-\infty$ (Theorem 4.5). In \$5 we prove the 
existence of ancient solutions on a special subfamily of fibre bundles over a product of an arbitrary number of QK manifolds (Theorem 5.6) and discuss their properties.

\section{Ricci flow on fibre bundles over products of two QK manifolds and its linearization at Einstein metrics}

In this section we derive a system of ODEs which is equivalent to the Ricci flow equation for a family of connection type metrics on certain $\frac{\mathrm{SO}(3) \times \cdots \times \mathrm{SO}(3))}{\Delta \mathrm{SO}(3)}$ fibre bundles over a product of $m$ arbitrary QK manifolds. We then specialize to the $m=2$ case, and determine the stationary solutions of the ODE system and establish properties of the linearization of the ODE system at these solutions which we will need in the later sections.

2.1. Some facts about QK manifolds. We first mention some standard facts about QK manifolds, referring the reader to [Be87] and [Sa89] for details. Let $\left(M^{4 n}, g\right), n \geq 2$, be a $\mathrm{QK}$ manifold, i.e., its holonomy group is contained in the subgroup $\mathrm{Sp}(n) \cdot \mathrm{Sp}(1)$ of $\mathrm{SO}(4 n)$. $(\mathrm{Sp}(n) \cdot \mathrm{Sp}(1)$ denotes more precisely the group $(\operatorname{Sp}(n) \times \operatorname{Sp}(1)) / \Delta \mathbb{Z}_{2}$, where $\Delta \mathbb{Z}_{2}$ denotes the diagonally embedded central subgroup.) We further assume that its holonomy group does not lie in $\operatorname{Sp}(n)$. Then it must be de Rham irreducible and Einstein with nonzero scalar curvature. A QK manifold with positive or negative scalar curvature will be called a positive or negative QK manifold, respectively. When $n=1$, it is customary to regard self-dual Einstein manifolds as the 4-dimensional analogs of QK manifolds. Those with positive scalar curvature are then known to be isometric to $S^{4}$ or $\mathbb{C P}^{2}$ by a celebrated theorem of N. Hitchin [Hi81]. We shall include the $n=1$ case in all of our discussions below.

To fix notation for this paper, let $\mathrm{Rc}_{g}=\Lambda g$ and let $\hat{P}$ denote the principal $\operatorname{Sp}(n) \cdot \operatorname{Sp}(1)$ holonomy bundle associated to the tangent bundle of $M$. The LeviCivita connection of $g$ induces a connection on $\hat{P}$, which upon projection induces a connection on the principal

$$
\left((\operatorname{Sp}(n) \times \operatorname{Sp}(1)) / \Delta \mathbb{Z}_{2}\right) / \operatorname{Sp}(n) \simeq \mathrm{SO}(3)
$$

bundle $P \doteqdot \hat{P} / \operatorname{Sp}(n)$ over $M$. This connection is in fact Yang-Mills, and has parallel curvature form [MS88. It is the analogue of the connection induced by the LeviCivita connection of a Kähler-Einstein manifold on the associated circle bundle of its anti-canonical line bundle. The $\mathrm{SO}(3)$ bundle $P$ is in general not spin, i.e., it does not in general admit a lift to an $\mathrm{SU}(2)$ bundle. Indeed, when $M$ is compact, it is spin precisely when $M$ is a quaternionic projective space.

Let $\left(M_{i}^{4 n_{i}}, g_{i}\right), i=1, \cdots, m$, be arbitrary QK manifolds with $\mathrm{Rc}_{g_{i}}=\Lambda_{i} g_{i}$. (Note that there is actually no need to assume that $\Lambda_{i}>0$ at this stage.) Let $P_{i} \rightarrow M_{i}$ be the principal $\mathrm{SO}(3)$ bundle described above that is associated to $\left(M_{i}, g_{i}\right)$. Let $\Delta \mathrm{SO}(3)$ be the diagonal subgroup of the product group $\mathrm{SO}(3) \times \cdots \times \mathrm{SO}(3)$. We define an

$$
\frac{\mathrm{SO}(3) \times \cdots \times \mathrm{SO}(3)}{\Delta \mathrm{SO}(3)}
$$

fibre bundle $\bar{P}$ of dimension $\left(\sum_{i=1}^{m} 4 n_{i}\right)+3(m-1)$ over $\bar{M} \doteqdot M_{1} \times \cdots \times M_{m}$ by

$$
\bar{P} \doteqdot\left(P_{1} \times \cdots \times P_{m}\right) \times \mathrm{SO}(3) \times \cdots \times \mathrm{SO}(3)\left(\frac{\mathrm{SO}(3) \times \cdots \times \mathrm{SO}(3)}{\Delta \mathrm{SO}(3)}\right) \rightarrow \bar{M}
$$


By the above discussion, each $P_{i}$ has a Yang-Mills connection whose curvature form is parallel. The product of these connections gives a connection on the $\mathrm{SO}(3) \times \cdots \times$ $\mathrm{SO}(3)$ principal bundle $P_{1} \times \cdots \times P_{m}$.

Using this connection on $P_{1} \times \cdots \times P_{m}$ and choosing vectors $\vec{a}=\left(a_{1}, \cdots, a_{m}\right)$ and $\vec{b}=\left(b_{1}, \cdots, b_{m}\right)$ with all positive components, we can construct a family of Riemannian metrics $\bar{g}_{\vec{a}, \vec{b}}$ on $\bar{P}$ uniquely determined by $\vec{a}$ and $\vec{b}$ satisfying the following conditions:

(B1) $\left(\bar{P}, \bar{g}_{\vec{a}, \vec{b}}\right) \rightarrow\left(\bar{M}, g_{\vec{b}}\right)$ is a Riemannian submersion with totally geodesic fibers, where $g_{\vec{b}} \doteqdot \sum_{i=1}^{m} b_{i} \pi_{i}^{*} g_{i}$ and $\pi_{i}: \bar{M} \rightarrow M_{i}$ is the natural projection map.

(B2) The restriction of $\bar{g}_{\vec{a}, \vec{b}}$ to the typical fiber in $\bar{P}$ is the normal homogeneous metric on $\frac{\mathrm{SO}(3) \times \cdots \times \mathrm{SO}(3)}{\Delta \mathrm{SO}(3)}$ induced from the bi-invariant metric $a_{1} B_{\mathfrak{s o}(3)} \oplus \cdots \oplus a_{m} B_{\mathfrak{s o}(3)}$ on $\mathrm{SO}(3) \times \cdots \times \mathrm{SO}(3)$ where $B_{\mathfrak{s o}(3)}(U, V)=-\operatorname{tr}(U V)$ for $U, V \in \mathfrak{s o}(3)$, the Lie algebra of $\mathrm{SO}(3)$.

Note that the isotropy representation of the fibre consists of $m-1$ copies of the adjoint representation of $\mathrm{SO}(3)$, so the space of invariant metrics on $\frac{\mathrm{SO}(3) \times \cdots \times \mathrm{SO}(3)}{\Delta \mathrm{SO}(3)}$ is given by

$$
\mathrm{GL}^{+}(m-1) / \mathrm{SO}(m-1) \equiv S_{+}^{2}\left(\mathbb{R}^{m-1}\right)
$$

with dimension $\frac{m(m-1)}{2}$. The invariant metrics on $\frac{\mathrm{SO}(3) \times \cdots \times \mathrm{SO}(3)}{\Delta \mathrm{SO}(3)}$ induced from biinvariant metrics on $\mathrm{SO}(3) \times \cdots \times \mathrm{SO}(3)$ as described in condition (B2) above is an $m$-dimensional subfamily except in the case $m=2$ (see Remark 2.2).

Remark 2.1. It should be mentioned that there is a conjecture, widely believed to be true, that a positive QK manifold must be isometric to a quaternionic symmetric space of compact type, also known as a Wolf space. In fact the 4- and 8-dimensional cases have been verified in [Hi81] and [PS91], respectively. A further rigidity result, valid for all dimensions, under the assumption of a non-vanishing second Betti number was obtained in LS94.

If the fundamental conjecture about positive QK manifolds is true, then each $M_{i}$ will have the form $G_{i} /\left(H_{i} \cdot \operatorname{Sp}(1)\right)$, where $G_{i}$ is a compact simple Lie group, and so $P_{i}=$ $G_{i} / H_{i}$. It follows that $\bar{P}$ and the corresponding Ricci flows are homogeneous. The status of the conjecture aside, we believe that the bundle formalism used in this paper may be more helpful for understanding ancient solutions beyond the homogeneous category. Furthermore, as we shall observe in Remark 3.2 , our analysis also leads to examples of ancient and immortal solutions of pseudo-Riemannian Ricci flows when some of the QK factors have negative scalar curvature.

2.2. Ricci flow on bundle $\bar{P}$ : an ODE system. Since the connection used to define our metrics $\bar{g}_{\vec{a}, \vec{b}}$ is Yang-Mills, it follows that the only nonzero components of the Ricci tensor of $\bar{g}_{\vec{a}, \vec{b}}$ come exclusively from the base or from the fibre, i.e., there are no cross terms involving both the fibre and the base. To derive the Ricci flow equations for the metrics $\bar{g}_{\vec{a}, \vec{b}}$ on $\bar{P}$, we use the computations in Wa92, particularly equations (2.1) and (2.2) there.

Consider a family of such Riemannian metrics $\bar{g}(\tau) \doteqdot \bar{g}_{\vec{a}(\tau), \vec{b}(\tau)}$, where $\tau \in \mathbb{R}$. First we consider tangential directions along the base. Let $\left\{X_{k}^{(i)}\right\}_{k=1}^{4 n_{i}}$ be an orthonormal 
frame in compact QK manifold $\left(M_{i}^{4 n_{i}}, g_{i}\right)$ and let $\left\{\tilde{X}_{k}^{(i)}\right\}_{k=1}^{4 n_{i}}$ be its horizontal lift to $\bar{P}$. Using [Wa92, (2.1)], it follows immediately that the part of the backwards Ricci flow equation involving the base directions is given by the equations

$$
\begin{aligned}
\frac{d b_{i}}{d \tau} & =\frac{d \bar{g}}{d \tau}\left(\tilde{X}_{k}^{(i)}, \tilde{X}_{k}^{(i)}\right)=2 \operatorname{Rc}_{\bar{g}}\left(\tilde{X}_{k}^{(i)}, \tilde{X}_{k}^{(i)}\right) \\
& =2 \Lambda_{i}-\frac{6 \Lambda_{i}^{2}}{\left(n_{i}+2\right)^{2}} \cdot \frac{a_{i}}{b_{i}}\left(1-\frac{a_{i}}{\hat{a}}\right), \quad i=1, \cdots, m,
\end{aligned}
$$

where $1 \leq k \leq 4 n_{i}$ is arbitrary but fixed and $\hat{a} \doteqdot \sum_{j=1}^{m} a_{j}$.

Next we consider the nonzero components of the Ricci tensor of $\bar{g}(\tau)$ coming from the fibre directions. Let $U$ be an element of Lie algebra $\mathfrak{s o}(3)$. For $i>j$ we define $\tilde{U}_{j i}=\left(0, \cdots, 0,-a_{i} U, 0, \cdots, 0, a_{j} U, 0, \cdots, 0\right)$ in which $-a_{i} U$ is at the $j$-th entry and $a_{j} U$ is at the $i$-th entry. For $i<j$ we define $\tilde{U}_{j i}=\left(0, \cdots, 0, a_{j} U, 0, \cdots, 0,-a_{i} U, 0, \cdots, 0\right)$ in which $a_{j} U$ is at the $i$-th entry and $-a_{i} U$ is at the $j$-th entry. Then

$$
\tilde{U}_{j i} \in \mathfrak{m}_{\vec{a}} \doteqdot\left\{\left(U_{1}, \cdots, U_{m}\right) \in \mathfrak{s o}(3) \times \cdots \times \mathfrak{s o}(3), \sum_{i=1}^{m} a_{i} U_{i}=0\right\}
$$

where $\mathfrak{m}_{\vec{a}}$ is the orthogonal complement of diagonal $\Delta \mathfrak{s o}(3)$ in $\mathfrak{s o}(3) \times \cdots \times \mathfrak{s o}(3)$ with respect to the bi-invariant metric $\oplus_{i=1}^{m} a_{i} B_{\mathfrak{s o}(3)}$. We divide the following discussion into two parts: $m \geq 3$ and $m=2$.

When $m \geq 3$, using [Wa92, (2.2)] we have that for distinct indices $i, j, k$

$$
\begin{aligned}
& \frac{d \bar{g}}{d \tau}\left(\tilde{U}_{k i}, \tilde{U}_{k j}\right)=\frac{d a_{k}}{d \tau} a_{i} a_{j} B(U, U), \\
& \operatorname{Rc}_{\bar{g}}\left(\tilde{U}_{k i}, \tilde{U}_{k j}\right)=\left(\frac{1}{4}+\frac{a_{k}}{2 \hat{a}}+\frac{2 n_{k} \Lambda_{k}^{2}}{\left(n_{k}+2\right)^{2}} \cdot \frac{a_{k}^{2}}{b_{k}^{2}}\right) a_{i} a_{j} B(U, U) .
\end{aligned}
$$

From the backwards Ricci flow equation $\frac{d \bar{g}}{d \tau}\left(\tilde{U}_{k i}, \tilde{U}_{k j}\right)=2 \operatorname{Rc}_{\bar{g}}\left(\tilde{U}_{k i}, \tilde{U}_{k j}\right)$ we have

$$
\frac{d a_{k}}{d \tau}=\frac{1}{2}+\frac{a_{k}}{\hat{a}}+\frac{4 n_{k} \Lambda_{k}^{2}}{\left(n_{k}+2\right)^{2}} \cdot \frac{a_{k}^{2}}{b_{k}^{2}}
$$

It follows that $\hat{a}$ satisfies the equation

$$
\frac{d \hat{a}}{d \tau}=\frac{m}{2}+1+\sum_{k=1}^{m} 4 n_{k} q_{k}^{2} \cdot \frac{a_{k}^{2}}{b_{k}^{2}}
$$

where $q_{k} \doteqdot \frac{\Lambda_{k}}{n_{k}+2}$. 
When $m=2$, the complement $\mathfrak{m}_{\vec{a}}$ equals $\left\{\tilde{U} \doteqdot\left(-a_{2} U, a_{1} U\right): U \in \mathfrak{s o}(3)\right\}$. By a computation similar to that in the $m \geq 3$ case we get the following formulas

$$
\begin{aligned}
\frac{d \bar{g}}{d \tau}(\tilde{U}, \tilde{U})= & \left(a_{2}^{2} \frac{d a_{1}}{d \tau}+a_{1}^{2} \frac{d a_{2}}{d \tau}\right) B(U, U), \\
\operatorname{Rc}_{\bar{g}}(\tilde{U}, \tilde{U})= & \left(\frac{1}{4}+\frac{a_{1}}{2\left(a_{1}+a_{2}\right)}+2 n_{1} q_{1}^{2} \cdot \frac{a_{1}^{2}}{b_{1}^{2}}\right) a_{2}^{2} B(U, U) \\
& +\left(\frac{1}{4}+\frac{a_{2}}{2\left(a_{1}+a_{2}\right)}+2 n_{2} q_{2}^{2} \cdot \frac{a_{2}^{2}}{b_{2}^{2}}\right) a_{1}^{2} B(U, U) .
\end{aligned}
$$

Let $\psi \doteqdot \frac{a_{1} a_{2}}{a_{1}+a_{2}}$. It follows from the backwards Ricci flow equation $\frac{d \bar{g}}{d \tau}(\tilde{U}, \tilde{U})=$ $2 \operatorname{Rc}_{\bar{g}}(\tilde{U}, \tilde{U})$ and the relation $\frac{d \psi}{d \tau}=\frac{\psi^{2}}{a_{1}^{2} a_{2}^{2}}\left(a_{2}^{2} \frac{d a_{1}}{d \tau}+a_{1}^{2} \frac{d a_{2}}{d \tau}\right)$ that

$$
\frac{d \psi}{d \tau}=\frac{1}{2}+4 n_{1} q_{1}^{2} \cdot \frac{\psi^{2}}{b_{1}^{2}}+4 n_{2} q_{2}^{2} \cdot \frac{\psi^{2}}{b_{2}^{2}} .
$$

On the other hand, if we set $a_{1}=a_{2}=2 \psi>0$ in the expressions of $\frac{d \bar{g}}{d \tau}(\tilde{U}, \tilde{U})$ and $\operatorname{Rc}_{\bar{g}}(\tilde{U}, \tilde{U})$ above, then the fibre part of the backwards Ricci flow equation becomes (2.7). There is no loss of generality to make this choice since the fibres are the irreducible symmetric space $\mathbb{R P}^{3}$ when $m=2$, and there is only one effective parameter for the space of invariant metrics.

Remark 2.2. In order to identify the effective parameters for the fibre metrics, one has to break the symmetry. Notice that the complement $\mathfrak{m}_{\vec{a}}$ to the diagonal subalgebra $\Delta \mathfrak{s o}(3)$ we chose depended on the biinvariant metric chosen. When $m=2$, it is natural to fix the complement to be the anti-diagonal $\mathfrak{m} \doteqdot\{(U,-U): U \in \mathfrak{s o}(3)\}$, which is the orthogonal complement when using $B_{\mathfrak{s o}(3)} \oplus B_{\mathfrak{s o}(3)}$ as the background metric. One checks easily that $a_{1} B_{\mathfrak{s o}(3)} \oplus a_{2} B_{\mathfrak{s o}(3)}$ and $a_{1}^{\prime} B_{\mathfrak{s o}(3)} \oplus a_{2}^{\prime} B_{\mathfrak{s o}(3)}$ induce the same metric on $\mathfrak{m}$ if and only if $a_{1}+a_{2}=a_{1}^{\prime}+a_{2}^{\prime}$.

Alternatively, we can also include $\mathfrak{s o}(3)$ into the first summand of $\mathfrak{s o}(3) \oplus \mathfrak{s o}(3)$ and project the image onto subspace $\mathfrak{m}_{\vec{a}}$ using $a_{1} B_{\mathfrak{s o}(3)} \oplus a_{2} B_{\mathfrak{s o}(3)}$. Then unit vector $U$ in $\left(\mathfrak{s o}(3), B_{\mathfrak{s o}(3)}\right)$ is mapped to $\frac{-1}{a_{1}+a_{2}}\left(-a_{2} U, a_{1} U\right)$, whose length squared equals to the parameter $\psi=a_{1} a_{2} /\left(a_{1}+a_{2}\right)$. This is what we used before in deriving our equations.

2.3. Properties of Einstein metrics on bundle $\bar{P}$ when $m=2$. Note that when $m=2, \bar{P}$ is an $\mathbb{R P}^{3} \approx \frac{\mathrm{SO}(3) \times \mathrm{SO}(3)}{\Delta \mathrm{SO}(3)}$ fibre bundle over $M_{1} \times M_{2}$. Let $\bar{g}(\tau)=\bar{g}_{\vec{a}(\tau), \vec{b}(\tau)}$ be a solution of the backwards Ricci flow on $\bar{P}$ given in $\$ 2.2$. It follows from (2.7) and (2.1) that $\psi(\tau)=\frac{a_{1} a_{2}}{a_{1}+a_{2}}, b_{1}(\tau)$ and $b_{2}(\tau)$ satisfy the following system:

$$
\begin{aligned}
& \frac{d \psi}{d \tau}=\frac{1}{2}+4 n_{1} q_{1}^{2} \cdot \frac{\psi^{2}}{b_{1}^{2}}+4 n_{2} q_{2}^{2} \cdot \frac{\psi^{2}}{b_{2}^{2}}, \\
& \frac{d b_{i}}{d \tau}=2\left(n_{i}+2\right) q_{i}-6 q_{i}^{2} \cdot \frac{\psi}{b_{i}}, \quad i=1,2,
\end{aligned}
$$

where $q_{i}=\frac{\Lambda_{i}}{n_{i}+2}$. By the discussion in Remark 2.2, these equations represent the backwards Ricci flow on $\bar{P}$ for the family of metrics in which $a_{1}(\tau)=a_{2}(\tau)=2 \psi(\tau)$. 
Remark 2.3. (i) The difference between the above equations and equations (3.2a) and $(3.2 \mathrm{~b})$ in [LW16] for circle bundles is the term $\frac{1}{2}$ in (2.8a). This term reflects that fact that the fibers $\mathbb{R P}^{3}$ now have constant positive curvature.

(ii) The difference between the above equations and equations (7.2) and (7.3) in BKN12 is that the base manifold $\bar{M}$ is reducible with two de Rham factors. Note that only the product of two Einstein manifolds with the same Einstein constants is Einstein. So the hypotheses in Proposition 7.1 in [BKN12] are seldom satisfied in the present situation.

To analyse the above system, as in [LW16, §3.1], we define new dependent variables $Y_{i} \doteqdot \frac{\psi}{b_{i}}, i=1,2$, and a new independent variable

$$
u=u(\tau) \doteqdot \int_{0}^{\tau} \frac{d \zeta}{\psi(\zeta)} .
$$

Using (2.8a) and (2.8b) we compute that

$$
\begin{aligned}
\frac{d Y_{i}}{d u} & =\psi \frac{d Y_{i}}{d \tau}=Y_{i} \frac{d \psi}{d \tau}-Y_{i}^{2} \frac{d b_{i}}{d \tau} \\
& =Y_{i}\left(\frac{1}{2}-2\left(n_{i}+2\right) q_{i} Y_{i}+6 q_{i}^{2} Y_{i}^{2}+4 n_{1} q_{1}^{2} Y_{1}^{2}+4 n_{2} q_{2}^{2} Y_{2}^{2}\right)
\end{aligned}
$$

Let $Y$ denote the vector $\left(Y_{1}, Y_{2}\right)$ and let

$$
\begin{aligned}
& E(Y) \doteqdot \frac{1}{2}+4 n_{1} q_{1}^{2} Y_{1}^{2}+4 n_{2} q_{2}^{2} Y_{2}^{2} \\
& F_{i}(Y) \doteqdot 2\left(n_{i}+2\right) q_{i} Y_{i}-6 q_{i}^{2} Y_{i}^{2}-E(Y), \quad i=1,2 .
\end{aligned}
$$

Equations (2.8a) and (2.8b) become

$$
\begin{aligned}
& \frac{1}{\psi} \frac{d \psi}{d u}=E(Y), \\
& \frac{d Y_{i}}{d u}=-Y_{i} F_{i}(Y), \quad i=1,2 .
\end{aligned}
$$

Given a solution $Y(u)$ of (2.10b),$\psi(u)$ is determined by (2.10a up to a multiplicative constant. By the relation $d \tau=\psi(\tau(u)) d u, \tau$ is recovered up to the same multiplicative constant since in (2.9) we arranged for $u=0$ to correspond to $\tau=0$. Then $b_{i}(u)$ can be found from $\frac{\psi}{Y_{i}}$, and we get $\left(\psi(\tau), b_{1}(\tau), b_{2}(\tau)\right)$ and the corresponding backwards flow solution $\bar{g}(\tau)$. Notice that a multiplicative constant actually amounts to a parabolic rescaling of $\bar{g}(\tau)$. Hence different integration constants do not lead to new solutions of the backwards Ricci flow. We have thus proved that each solution $Y(u)$ gives rise to a single solution $\bar{g}(\tau)$ of the backwards Ricci flow. Therefore we will focus the solutions of (2.10b) from now on.

As a preliminary step we have the following lemma about the stationary solutions, two of which correspond to Einstein metrics on $\bar{P}$. For the rest of this section we will assume that $n_{1} \leq n_{2}$ (by interchanging $M_{1}$ and $M_{2}$ if necessary).

Lemma 2.4. The zeros of vector field $\left(-Y_{1} F_{1}(Y),-Y_{2} F_{2}(Y)\right)$ are

(i) the origin,

(ii) the points $v_{1}=\left(\frac{1}{\left(4 n_{1}+6\right) q_{1}}, 0\right), \tilde{v}_{1}=\left(\frac{1}{2 q_{1}}, 0\right), v_{2}=\left(0, \frac{1}{\left(4 n_{2}+6\right) q_{2}}\right), \tilde{v}_{2}=\left(0, \frac{1}{2 q_{2}}\right)$, 
(iii) the Einstein points $\xi=\left(\xi_{1}, \xi_{2}\right)$ and $\eta=\left(\eta_{1}, \eta_{2}\right)$. We further have $0<\eta_{1}<\xi_{1}$ and $0<\eta_{2}<\xi_{2}$.

Proof. (i) If both $Y_{1}$ and $Y_{2}$ are zero, we get the origin.

(ii) This occurs when exactly one of $Y_{1}$ or $Y_{2}$ is zero. If $Y_{2}=0$, then $F_{1}\left(Y_{1}, 0\right)=0$. We observe that equation

$$
F_{1}(Y)=-\left(4 n_{1}+6\right) q_{1}^{2} Y_{1}^{2}+2\left(n_{1}+2\right) q_{1} Y_{1}-4 n_{2} q_{2}^{2} Y_{2}^{2}-\frac{1}{2}=0
$$

represents an ellipse in $Y$-space with center at $\left(\frac{n_{1}+2}{\left(4 n_{1}+6\right) q_{1}}, 0\right)$. The ellipse intersects the $Y_{1}$-axis at $v_{1}=\left(\frac{1}{\left(4 n_{1}+6\right) q_{1}}, 0\right)$ and $\tilde{v}_{1}=\left(\frac{1}{2 q_{1}}, 0\right)$, i.e., solutions of the equation $F_{1}\left(Y_{1}, 0\right)=0$. So for points lying in the ellipse we have $\frac{1}{\left(4 n_{1}+6\right) q_{1}} \leq Y_{1} \leq \frac{1}{2 q_{1}}$ and $\left|Y_{2}\right| \leq \frac{n_{1}+1}{2 q_{2} \sqrt{n_{2}\left(4 n_{1}+6\right)}}$.

Likewise, if $Y_{1}=0$, then $F_{2}\left(0, Y_{2}\right)=0 . F_{2}(Y)=0$ is an ellipse with center at $\left(0, \frac{n_{2}+2}{\left(4 n_{2}+6\right) q_{2}}\right)$ and whose intersection with the $Y_{2}$-axis occur at $v_{2}=\left(0, \frac{1}{\left(4 n_{2}+6\right) q_{2}}\right)$ and $\tilde{v}_{2}=\left(0, \frac{1}{2 q_{2}}\right)$. So for points lying in the ellipse we have $\frac{1}{\left(4 n_{2}+6\right) q_{2}} \leq Y_{2} \leq \frac{1}{2 q_{2}}$ and $\left|Y_{1}\right| \leq \frac{n_{2}+1}{2 q_{1} \sqrt{n_{1}\left(4 n_{2}+6\right)}}$.

(iii) If both $Y_{1}$ and $Y_{2}$ are non-zero, we get $F_{1}(Y)=F_{2}(Y)=0$. Now we consider the relation between the solutions of equations $F_{1}(Y)=F_{2}(Y)=0$ and the Einstein metrics on $\bar{P}$ of the form $\bar{g}_{\vec{a}, \vec{b}}$. We have

$$
2\left(n_{1}+2\right) q_{1} Y_{1}-6 q_{1}^{2} Y_{1}^{2}=E(Y)=2\left(n_{2}+2\right) q_{2} Y_{2}-6 q_{2}^{2} Y_{2}^{2} .
$$

Set $E(Y) \doteqdot 2 \Lambda \psi$ for some $\Lambda>0$. Hence for $i=1,2$,

$$
\frac{1}{4}+\frac{2 n_{1} \Lambda_{1}^{2}}{\left(n_{1}+2\right)^{2}} Y_{1}^{2}+\frac{2 n_{2} \Lambda_{2}^{2}}{\left(n_{2}+2\right)^{2}} Y_{2}^{2}=\Lambda \psi=\Lambda_{i} Y_{i}-\frac{3 \Lambda_{i}^{2}}{\left(n_{i}+2\right)^{2}} Y_{i}^{2} .
$$

So $\psi$ and $b_{i}$ satisfy the equations for an Einstein metric ([Wa92, (2.3)]) and we have $\Lambda=\frac{\Lambda_{i}}{b_{i}}-\frac{3 \Lambda_{i}^{2}}{\left(n_{i}+2\right)^{2}} \cdot \frac{\psi}{b_{i}^{2}}$. It is known from [Wa92, p.313] that there are exactly two solutions $Y=\xi=\left(\xi_{1}, \xi_{2}\right)$ and $Y=\eta=\left(\eta_{1}, \eta_{2}\right)$ of the above equations which represent Einstein metrics on $\bar{P}$.

To deduce the inequalities in (iii), let

$$
y_{0} \doteqdot y_{0}(Y) \doteqdot \frac{1}{2 E(Y)}, \quad y_{i} \doteqdot y_{i}(Y) \doteqdot 4\left(n_{i}+2\right) q_{i} y_{0}(Y) Y_{i}, \quad i=1,2
$$

Then the equations $F_{1}(Y)=F_{2}(Y)=0$ together with the definition of $E(Y)$ give rise to an equivalent system of equations for the variables $y_{0}, y_{1}, y_{2}$,

$$
\begin{aligned}
& 3\left(y_{0}-1\right)+2 n_{1}\left(y_{1}-1\right)+2 n_{2}\left(y_{2}-1\right)=0, \\
& y_{0}=\frac{3}{4\left(n_{i}+2\right)^{2}} \cdot \frac{y_{i}^{2}}{y_{i}-1}, \quad i=1,2 .
\end{aligned}
$$


Actually (2.13b) follows from (2.11) and (2.13a) follows from (2.12) and (2.13b). Since we assume $n_{1} \leq n_{2}$, the two Einstein solutions of the system satisfy ([Wa92, p.313])

$$
y_{0} \in\left[\frac{3}{\left(n_{1}+2\right)^{2}}, 1\right), \quad 1<y_{2} \leq y_{1} \leq 2 .
$$

Hence by solving the quadratic equation (2.13b) for $y_{i}$ we get

$$
y_{i}=y_{i}\left(y_{0}\right)=\frac{2\left(n_{i}+2\right)^{2} y_{0}}{3}\left(1-\sqrt{1-\frac{3}{y_{0}} \cdot \frac{1}{\left(n_{i}+2\right)^{2}}}\right),
$$

in which we took the negative sign in the quadratic formula because of $y_{i} \leq 2$. Hence we can define a function $\phi$ of $y_{0} \in\left[\frac{3}{\left(n_{1}+2\right)^{2}}, 1\right)$

$$
\phi\left(y_{0}\right) \doteqdot 3\left(y_{0}-1\right)+2 n_{1}\left(y_{1}\left(y_{0}\right)-1\right)+2 n_{2}\left(y_{2}\left(y_{0}\right)-1\right) \text {. }
$$

Note that $\phi$ is actually a convex function (see [Wa92, p.313]) which accounts for the existence of the two Einstein points $\xi$ and $\eta$.

Let $\left(y_{01}, y_{11}, y_{21}\right)$ (corresponding to $\left.\xi\right)$ and $\left(y_{02}, y_{12}, y_{22}\right)$ (corresponding to $\eta$ ) be the two solutions of (2.13a) and (2.13b) with $y_{01}<y_{02}$. Then

$$
\begin{aligned}
\xi_{1} & =\frac{y_{11}}{4 \Lambda_{1} y_{01}}=\frac{\left(n_{1}+2\right)^{2}}{6 \Lambda_{1}}\left(1-\sqrt{1-\frac{3}{y_{01}} \cdot \frac{1}{\left(n_{1}+2\right)^{2}}}\right) \\
> & \frac{\left(n_{1}+2\right)^{2}}{6 \Lambda_{1}}\left(1-\sqrt{1-\frac{3}{y_{02}} \cdot \frac{1}{\left(n_{1}+2\right)^{2}}}\right)=\eta_{1} .
\end{aligned}
$$

Similarly we have $\eta_{2}<\xi_{2}$. This proves (iii) and hence the lemma.

Next we give some estimates of $\eta$ which will be used later to study its linear stability as a solution of (2.10b).

Lemma 2.5. The Einstein point $\eta=\left(\eta_{1}, \eta_{2}\right)$ satisfies the following properties:

(i) When $n_{2} \geq n_{1} \geq 2$ and $\left(n_{1}, n_{2}\right) \neq(2,2),(2,3)$, we have $q_{i} \eta_{i}<\frac{0.4661}{n_{i}+2}$ for $i=1,2$.

(ii) When $n_{1}=1$ and $n_{2} \geq 2$ we have $q_{1} \eta_{1}<0.1608$ and $q_{2} \eta_{2}<\frac{0.4661}{n_{2}+2}$.

(iii) When $\left(n_{1}, n_{2}\right)=(2,2)$ we have $q_{1} \eta_{1}<0.0912$ and $q_{2} \eta_{2}<0.0912$.

(iv) When $\left(n_{1}, n_{2}\right)=(2,3)$ we have $q_{1} \eta_{1}<0.1204$ and $q_{2} \eta_{2}<0.0928$.

(v) When $\left(n_{1}, n_{2}\right)=(1,1)$ we have $q_{1} \eta_{1}<0.1303$ and $q_{2} \eta_{2}<0.1303$.

Proof. We will divide the proof into five cases. In each case we find an appropriate $y_{0}^{*}$ such that $\phi\left(y_{0}^{*}\right)<0$. Since $y_{02}$ is the larger of the two solutions of $\phi(y)=0$, it follows that $y_{02}>y_{0}^{*}$. Hence $y_{i 2}\left(y_{02}\right)<y_{i 2}\left(y_{0}^{*}\right)$ for $i=1,2$ by (2.14) and the desired estimates will follow.

If we choose $y_{0}^{*}=\frac{6+\sqrt{2}}{12}$, then for all $n_{i} \geq 1, i=1,2$,

$$
\frac{3}{y_{0}^{*}} \cdot \frac{1}{\left(n_{i}+2\right)^{2}}<2(\sqrt{2}-1) \text {. }
$$

Note that

$$
1-\frac{1}{2} \alpha-\frac{1}{4} \alpha^{2}<\sqrt{1-\alpha} \text { for } \alpha \in(0,2(\sqrt{2}-1)) \text {. }
$$


Applying this inequality to (2.14) with $\alpha=\frac{3}{y_{0}^{*}} \cdot \frac{1}{\left(n_{i}+2\right)^{2}}$ we can estimate $\phi\left(y_{0}^{*}\right)$ as follows:

$$
\begin{aligned}
\phi\left(y_{0}^{*}\right) & <3\left(y_{0}^{*}-1\right)+\frac{3}{y_{0}^{*}}\left(\frac{n_{1}}{\left(n_{1}+2\right)^{2}}+\frac{n_{2}}{\left(n_{2}+2\right)^{2}}\right) \\
& =3(6-\sqrt{2}) \cdot\left(-\frac{1}{12}+\frac{6}{17} \cdot\left(\frac{n_{1}}{\left(n_{1}+2\right)^{2}}+\frac{n_{2}}{\left(n_{2}+2\right)^{2}}\right)\right) \\
& \leq 0
\end{aligned}
$$

for $n_{2} \geq n_{1} \geq 2$ and $n_{2} \geq 4$, or $\left(n_{1}, n_{2}\right)=\left(1, n_{2}\right)$ for any $n_{2} \geq 2$. By (2.14) and a direct computation we also have $\phi\left(y_{0}^{*}\right)<0$ for $\left(n_{1}, n_{2}\right)=(3,3)$. Hence for these $\left(n_{1}, n_{2}\right)$ the corresponding

$$
y_{02}>y_{0}^{*}=\frac{6+\sqrt{2}}{12} .
$$

There are now five cases to consider.

(i) $n_{2} \geq n_{1} \geq 2$ and $\left(n_{1}, n_{2}\right) \neq(2,2),(2,3)$ : By the definition of $\eta_{i}$, (2.14), (2.20), and (2.18) $)$ we get for $y_{0}^{*}=\frac{6+\sqrt{2}}{12}$

$$
\begin{aligned}
q_{i} \eta_{i} & =\frac{n_{i}+2}{6}\left(1-\sqrt{1-\frac{3}{y_{02}} \cdot \frac{1}{\left(n_{i}+2\right)^{2}}}\right) \\
& <\frac{n_{i}+2}{6}\left(1-\sqrt{1-\frac{3}{y_{0}^{*}} \cdot \frac{1}{\left(n_{i}+2\right)^{2}}}\right) \\
& <\frac{1}{n_{i}+2} \cdot\left(\frac{1}{4 y_{0}^{*}}+\frac{3}{8\left(y_{0}^{*}\right)^{2}\left(n_{i}+2\right)^{2}}\right) \\
& <\frac{0.4661}{n_{i}+2} .
\end{aligned}
$$

Note that (2.21) holds whenever $y_{02}>y_{0}^{*}$.

(ii) $n_{1}=1$ and $n_{2} \geq 2$ : By the definition of $\eta_{i}$, (2.20) and (2.21) we have for $y_{0}^{*}=\frac{6+\sqrt{2}}{12}$

$$
\begin{aligned}
& q_{1} \eta_{1}<\frac{1}{2}\left(1-\sqrt{1-\frac{1}{3 y_{0}^{*}}}\right)<0.1608, \text { and } \\
& q_{2} \eta_{2}<\frac{0.4661}{n_{i}+2} .
\end{aligned}
$$

(iii) $\left(n_{1}, n_{2}\right)=(2,2)$ : Taking $y_{0}^{*}=\frac{3+\sqrt{2}}{6}$, we use (2.14) to compute that

$$
y_{1}\left(y_{0}^{*}\right)=y_{2}\left(y_{0}^{*}\right)<1.0732 .
$$

Hence we get $\phi\left(y_{0}^{*}\right)<0$ and $y_{02}>y_{0}^{*}=\frac{3+\sqrt{2}}{6}$. We then apply (2.21) to estimate

$$
q_{i} \eta_{i}<\frac{2}{3}\left(1-\sqrt{1-\frac{1}{y_{0}^{*}} \cdot \frac{3}{16}}\right)<0.0912, \quad i=1,2 .
$$


(iv) $\left(n_{1}, n_{2}\right)=(2,3)$ : We take $y_{0}^{*}=\frac{10+\sqrt{2}}{20}$. Then for this $y_{0}^{*}$ both (2.17) and (2.19) hold for $\left(n_{1}, n_{2}\right)=(2,3)$, hence $y_{02}>y_{0}^{*}=\frac{10+\sqrt{2}}{20}$. By (2.21) we have

$$
\begin{aligned}
& q_{1} \eta_{1}<\frac{2}{3}\left(1-\sqrt{1-\frac{1}{y_{0}^{*}} \cdot \frac{3}{16}}\right)<0.1204 \\
& q_{2} \eta_{2}<\frac{5}{6}\left(1-\sqrt{1-\frac{1}{y_{0}^{*}} \cdot \frac{3}{25}}\right)<0.0928 .
\end{aligned}
$$

(v) $\left(n_{1}, n_{2}\right)=(1,1)$ : We take $y_{0}^{*}=\frac{3+\sqrt{2}}{6}$. Then $y_{1}\left(y_{0}^{*}\right)=y_{2}\left(y_{0}^{*}\right)<1.1498$ and $\phi\left(y_{0}^{*}\right)<-0.1936<0$. Hence $y_{02}>y_{0}^{*}=\frac{3+\sqrt{2}}{6}$ and

$$
q_{i} \eta_{i}<\frac{1}{2}\left(1-\sqrt{1-\frac{1}{3 y_{0}^{*}}}\right)<0.1303 .
$$

This completes the proof of the lemma.

2.4. Linearization of ODEs (2.10b) . We can now consider the linear stability of the vector field $\left(-Y_{1} F_{1}(Y),-Y_{2} F_{2}(Y)\right)$ at each of its zeros given in Lemma 2.4.

Proposition 2.6. For the vector field $\left(-Y_{1} F_{1}(Y),-Y_{2} F_{2}(Y)\right)$

(i) $(0,0)$ is a source;

(ii) $v_{1}$ is a hyperbolic point with stable eigen-direction (1,0) and unstable eigendirection $(0,1)$;

(iii) $v_{2}$ is a hyperbolic point with unstable eigen-direction $(1,0)$ and stable eigendirection $(0,1)$;

(iv) $\tilde{v}_{1}$ is a source;

(v) $\tilde{v}_{2}$ is a source;

(vi) $\eta$ is a sink; and

(vii) $\xi$ is a hyperbolic point.

Proof. The Jacobian of the vector field $\left(-Y_{1} F_{1}(Y),-Y_{2} F_{2}(Y)\right)$ is given by

$$
\mathcal{L}_{Y} \doteqdot\left(\begin{array}{cc}
h_{1}(Y) & 8 n_{2} q_{2}^{2} Y_{1} Y_{2} \\
8 n_{1} q_{1}^{2} Y_{1} Y_{2} & h_{2}(Y)
\end{array}\right)
$$

where

$$
\begin{aligned}
& h_{1}(Y) \doteqdot-4\left(n_{1}+2\right) q_{1} Y_{1}+3\left(4 n_{1}+6\right) q_{1}^{2} Y_{1}^{2}+4 n_{2} q_{2}^{2} Y_{2}^{2}+\frac{1}{2} \\
& h_{2}(Y) \doteqdot-4\left(n_{2}+2\right) q_{2} Y_{2}+3\left(4 n_{2}+6\right) q_{2}^{2} Y_{2}^{2}+4 n_{1} q_{1}^{2} Y_{1}^{2}+\frac{1}{2}
\end{aligned}
$$

(i) At $Y=(0,0)$ we have

$$
\mathcal{L}_{(0,0)}=\left(\begin{array}{cc}
\frac{1}{2} & 0 \\
0 & \frac{1}{2}
\end{array}\right)
$$

Hence $(0,0)$ is a source. 
(ii) At $Y=v_{1}$ we have

$$
\mathcal{L}_{v_{1}}=\left(\begin{array}{cc}
-\frac{n_{1}+1}{2 n_{1}+3} & 0 \\
0 & \frac{n_{1}}{\left(2 n_{1}+3\right)^{2}}+\frac{1}{2}
\end{array}\right) .
$$

Hence $v_{1}$ is a hyperbolic point. It is clear that $(1,0)$ is the stable eigen-direction and $(0,1)$ is the unstable eigen-direction.

(iii) This follows from its symmetry to case (ii).

(iv) At $Y=\tilde{v}_{1}$ we have

$$
\mathcal{L}_{\tilde{v}_{1}}=\left(\begin{array}{cc}
n_{1}+1 & 0 \\
0 & n_{1}+\frac{1}{2}
\end{array}\right)
$$

Hence $\tilde{v}_{1}$ is a source.

(v) This follows from its symmetry to case (iv).

(vi) At $Y=\eta$, using $F_{1}(\eta)=F_{2}(\eta)=0$, we have

$$
h_{1}(\eta)=\left(8 n_{1}+6\right) q_{1}^{2} \eta_{1}^{2}-E(\eta), \quad h_{2}(\eta)=\left(8 n_{2}+6\right) q_{2}^{2} \eta_{2}^{2}-E(\eta),
$$

and $\mathcal{L}_{\eta}=-E(\eta) I+\beta_{\eta}$ where for $\chi=\left(\chi_{1}, \chi_{2}\right)$

$$
\beta_{\chi} \doteqdot\left(\begin{array}{cc}
\left(8 n_{1}+6\right) q_{1}^{2} \chi_{1}^{2} & 8 n_{2} q_{2}^{2} \chi_{1} \chi_{2} \\
8 n_{1} q_{1}^{2} \chi_{1} \chi_{2} & \left(8 n_{2}+6\right) q_{2}^{2} \chi_{2}^{2}
\end{array}\right) .
$$

We need to analyze the eigenvalues of $\beta_{\eta}$.

Suppose $\chi_{1}>0$ and $\chi_{2}>0$. Using the diagonal matrix $\left[\chi_{1}, \chi_{2}\right]$, we compute that

$$
\alpha_{\chi} \doteqdot\left[\chi_{1}^{-1}, \chi_{2}^{-1}\right] \cdot \beta_{\chi} \cdot\left[\chi_{1}, \chi_{2}\right]=\left(\begin{array}{cc}
\left(8 n_{1}+6\right) q_{1}^{2} \chi_{1}^{2} & 8 n_{2} q_{2}^{2} \chi_{2}^{2} \\
8 n_{1} q_{1}^{2} \chi_{1}^{2} & \left(8 n_{2}+6\right) q_{2}^{2} \chi_{2}^{2}
\end{array}\right) \text {. }
$$

Note that since $\operatorname{det} \alpha_{\chi}>0$, we may assume that $\rho_{1}(\chi) \geq \rho_{2}(\chi)>0$ are the two eigenvalues of $\alpha_{\chi}$. Then the eigenvalues of $\mathcal{L}_{\chi}$ are given by $-E(\chi)+\rho_{i}(\chi)$.

A simple calculation of the eigenvalues of $\alpha_{\eta}$ using the Einstein condition gives

$$
-E(\eta)+\rho_{1}(\eta)=3 q_{1}^{2} \eta_{1}^{2}+3 q_{2}^{2} \eta_{2}^{2}-\frac{1}{2}+\sqrt{A(\eta)},
$$

where

$$
\begin{aligned}
A(\chi) \doteqdot & \left(4 n_{1}+3\right)^{2} q_{1}^{4} \chi_{1}^{4}+\left(4 n_{2}+3\right)^{2} q_{2}^{4} \chi_{2}^{4} \\
& +\left(32 n_{1} n_{2}-24 n_{1}-24 n_{2}-18\right) q_{1}^{2} q_{2}^{2} \chi_{1}^{2} \chi_{2}^{2} .
\end{aligned}
$$

It is easy to see that

$$
A(\eta)<\left(\left(4 n_{1}+3\right) q_{1}^{2} \eta_{1}^{2}+\left(4 n_{2}+3\right) q_{2}^{2} \eta_{2}^{2}\right)^{2} .
$$

So for all $n_{2} \geq n_{1} \geq 1$

$$
-E(\eta)+\rho_{1}(\eta)<\left(4 n_{1}+6\right) q_{1}^{2} \eta_{1}^{2}+\left(4 n_{2}+6\right) q_{2}^{2} \eta_{2}^{2}-\frac{1}{2} .
$$

Hence by Lemma 2.5(i) we have

$$
-E(\eta)+\rho_{1}(\eta)<\left(4 n_{1}+6\right)\left(\frac{0.4661}{n_{1}+2}\right)^{2}+\left(4 n_{2}+6\right)\left(\frac{0.4661}{n_{2}+2}\right)^{2}-\frac{1}{2}<0
$$


when $n_{2} \geq n_{1} \geq 2$ and $\left(n_{1}, n_{2}\right) \neq(2,2),(2,3)$. By Lemma 2.5 (ii) we have for $n_{1}=1$ and $n_{2} \geq 2$

$$
-E(\eta)+\rho_{1}(\eta)<10 \cdot(0.1608)^{2}+\left(4 n_{2}+6\right) \cdot\left(\frac{0.4661}{n_{2}+2}\right)^{2}-\frac{1}{2}<0 .
$$

When $\left(n_{1}, n_{2}\right)=(2,2)$, Lemma 2.5)(iii) we have

$$
-E(\eta)+\rho_{1}(\eta)<14 \cdot(0.0912)^{2}+14 \cdot(0.0912)^{2}-\frac{1}{2}<0 .
$$

When $\left(n_{1}, n_{2}\right)=(2,3)$, by Lemma 2.5)(iv) we have

$$
-E(\eta)+\rho_{1}(\eta)<14 \cdot(0.1204)^{2}+18 \cdot(0.0928)^{2}-\frac{1}{2}<0 .
$$

When $\left(n_{1}, n_{2}\right)=(1,1)$, by Lemma 2.5)(v) we have

$$
-E(\eta)+\rho_{1}(\eta)<10 \cdot(0.1303)^{2}+10 \cdot(0.1303)^{2}-\frac{1}{2}<0 .
$$

We have therefore proved that $-E(\eta)+\rho_{1}(\eta)<0$ for all $\left(n_{1}, n_{2}\right)$. Hence $\eta$ is a sink.

(vii) Similar to case (vi) we have $\mathcal{L}_{\xi}=-E(\xi) I+\beta_{\xi}$ where $\beta_{\xi}$ is obtained from (2.24) by setting $\chi=\xi$. For the corresponding matrix $\alpha_{\xi}$ defined by (2.25) with eigenvalues $\rho_{1}(\xi) \geq \rho_{2}(\xi)>0$, we obtain $-E(\xi)+\rho_{1}(\xi) \geq-E(\xi)+\rho_{2}(\xi)$ for the two eigenvalues of $\mathcal{L}_{\xi}$.

To see that $-E(\xi)+\rho_{2}(\xi)<0$, it is easy to check that for all $n_{1}, n_{2}, q_{1} \xi_{1}, q_{2} \xi_{2}$,

$$
A(\xi)>\left(3 q_{1}^{2} \xi_{1}^{2}+3 q_{2}^{2} \xi_{2}^{2}\right)^{2}
$$

from which it follows that

$$
-E(\xi)+\rho_{2}(\xi)=3 q_{1}^{2} \xi_{1}^{2}+3 q_{2}^{2} \xi_{2}^{2}-\frac{1}{2}-\sqrt{A(\xi)}<0
$$

Note that in the line above we took the negative square root in front of $\sqrt{A(\xi)}$ since $\rho_{2}(\xi)$ is by choice the smaller eigenvalue.

We will give an indirect proof that $-E(\xi)+\rho_{1}(\xi)>0$ when we establish the second claim of Proposition 3.3(ii). Hence $\xi$ is a hyperbolic point. In view of this the proof of Proposition 2.6] is complete.

\section{Ancient solutions on $\mathbb{R P}^{3}$ fibre bundles}

In this section we first prove the existence of ancient solutions on the $\mathbb{R P}^{3}$ fibre bundles $\bar{P}$ over a product of two QK manifolds. Then we turn to study the asymptotic behaviors of the metric tensors.

3.1. Existence of ancient solutions on $\mathbb{R P}^{3}$ fibre bundles. We shall consider two regions in $\mathbb{R}^{2}$ : a convex region

$$
\Omega_{1} \doteqdot\left\{Y: F_{1}(Y) \geq 0 \text { and } F_{2}(Y) \geq 0\right\}
$$

and a (non-convex) region

$$
\Omega_{2} \doteqdot \text { bounded component of }\left\{Y: Y_{1} \geq 0, Y_{2} \geq 0, F_{1}(Y) \leq 0 \text {, and } F_{2}(Y) \leq 0\right\} \text {. }
$$


Theorem 3.1. Suppose $m=2$ and the $Q K$ manifolds $\left(M_{i}^{4 n_{i}}, g_{i}\right)$ have positive scalar curvature, i.e., $\Lambda_{i}>0$ for $i=1,2$. Then the ODE system (2.8a) and (2.8b) has the following types of long-time solutions $\left(\psi(\tau), b_{1}(\tau), b_{2}(\tau)\right)$ with $0 \leq \tau<+\infty$ corresponding to the solutions $Y(u)$ of (2.10b) :

(i) There is a continuous 1-parameter family of solutions $Y(u)$ with $\lim _{u \rightarrow \infty} Y(u)=\eta$ and a corresponding continuous 1-parameter family of ancient solutions $\bar{g}(\tau)$ of the Ricci flow on $\bar{P}$. Furthermore, as special cases,

(ia) if the initial data $Y(0)=\left(\frac{\psi(0)}{b_{1}(0)}, \frac{\psi(0)}{b_{2}(0)}\right) \in \Omega_{1}$, then the solution $Y(u)$ stays in $\Omega_{1}$ and $\lim _{u \rightarrow \infty} Y(u)=\eta$;

(ib) if the initial data $Y(0)=\left(\frac{\psi(0)}{b_{1}(0)}, \frac{\psi(0)}{b_{2}(0)}\right) \in \Omega_{2}$, then the solution $Y(u)$ stays in $\Omega_{2}$ $\lim _{u \rightarrow \infty} Y(u)=\eta$.

(ii) There are two distinct solutions $Y(u)$ with $\lim _{u \rightarrow \infty} Y(u)=\xi$ and correspondingly two distinct ancient solutions $\bar{g}(\tau)$ on $\bar{P}$.

Proof. (i) By Proposition 2.6(vi) the local stable manifold at $\eta$ has dimension 2, so the existence of a continuous 1-parameter family of ancient solutions $Y(u)$ follows from the Hartman-Grobman Theorem.

From (2.10a) we can conclude that $\psi(u)$ exists for all $u \in[0, \infty)$ and that $\psi(u)^{-1}$ is bounded from above. Hence from (2.9) we get $\tau(u) \rightarrow \infty$ when $u \rightarrow \infty$. In view of the discussion after (2.10b), we get a continuous 1-parameter family of ancient solutions $\left(\psi(\tau), b_{1}(\tau), b_{2}(\tau)\right)$. This in turn gives a continuous 1-parameter family of ancient solutions $\bar{g}(\tau)$ (modulo time translation and parabolic scaling).

(ia) Consider a solution $Y(u)$ with $Y(0) \in \Omega_{1} \backslash\{\xi, \eta\}$. If at some time $u_{0} \geq 0$ we have $Y\left(u_{0}\right) \in \partial \Omega_{1} \backslash\{\xi, \eta\}$, then either $F_{1}\left(Y\left(u_{0}\right)\right)=0$ or $F_{2}\left(Y\left(u_{0}\right)\right)=0$. In the first case, computing at $Y\left(u_{0}\right)$, we obtain

$$
\nabla F_{1}=\left(2\left(n_{1}+2\right) q_{1}-2\left(4 n_{1}+6\right) q_{1}^{2} Y_{1},-8 n_{2} q_{2}^{2} Y_{2}\right)
$$

so that

$$
\nabla F_{1} \cdot\left(0,-Y_{2} F_{2}(Y)\right)=8 n_{2} q_{2}^{2} Y_{2}^{2} F_{2}(Y)>0,
$$

where the last inequality follows from the assumption that $Y\left(u_{0}\right) \neq \xi$ or $\eta$. Similarly, if $F_{2}\left(Y\left(u_{0}\right)\right)=0$, we have $\nabla F_{2} \cdot\left(-Y_{1} F_{1}(Y), 0\right)>0$. Hence the solution $Y(u)$ stays in $\Omega_{1}$ for all $u$. The property $\lim _{u \rightarrow \infty} Y(u)=\eta$ will be proved in Theorem 3.3 below.

(ib) The vector field $-\left(Y_{1} F_{1}(Y), Y_{2} F_{2}(Y)\right)$ has 4 zeros $\left\{0, \eta, v_{1}, v_{2}\right\}$ in $\Omega_{2}$. Take a solution $Y(u)$ of (2.10b) with $Y(0) \in \Omega_{2} \backslash\left\{0, \eta, v_{1}, v_{2}\right\}$. If there is a time $u_{0}$ at which the solution $Y(u)$ hits the boundary where $F_{1}(Y)=0$, then computing at $Y\left(u_{0}\right)$ as above one gets

$$
\nabla F_{1} \cdot\left(0,-Y_{2} F_{2}(Y)\right)=8 n_{2} q_{2}^{2} Y_{2}^{2} F_{2}(Y) \leq 0
$$

with equality iff we are at the fixed points $\eta$ or $v_{1}$. If the solution $Y(u)$ hits the boundary where $F_{2}(Y)=0$ at time $u_{0}$, we have at $Y\left(u_{0}\right)$

$$
\nabla F_{2} \cdot\left(-Y_{1} F_{1}(Y), 0\right)=8 n_{1} q_{1}^{2} Y_{1}^{2} F_{1}(Y) \leq 0
$$

with equality iff we are at the fixed points $\eta$ or $v_{2}$.

If the solution $Y(u)$ hits the part of the boundary where $Y_{2}=0$ at time $u_{0}$, then we have $F_{1}\left(Y\left(u_{0}\right)\right) \leq 0$ and $F_{2}\left(Y\left(u_{0}\right)\right)<0$, so that

$$
(0,1) \cdot\left(-Y_{1} F_{1}(Y),-Y_{2} F_{2}(Y)\right)=0 .
$$


Finally, if the solution $Y(u)$ hits the boundary where $Y_{1}=0$ at time $u_{0}$, then we have $F_{1}\left(Y\left(u_{0}\right)\right)<0$ and $F_{2}\left(Y\left(u_{0}\right)\right) \leq 0$. Hence

$$
(1,0) \cdot\left(-Y_{1} F_{1}(Y),-Y_{2} F_{2}(Y)\right)=0 .
$$

Combining the above analysis we conclude that $Y(u)$ remains in $\Omega_{2}$ for all $u \geq 0$. The property $\lim _{u \rightarrow \infty} Y(u)=\eta$ will be proved in Theorem 3.4 below.

(ii) The existence of two distinct solutions $Y(u)$ follows from the Hartman-Grobman Theorem since the local stable manifold at $\xi$ has dimension 1. By analogous arguments to those for case (i) we obtain the existence of two ancient solutions $\bar{g}(\tau)$. This proves case (ii) and hence also completes the proof of the Theorem.

Remark 3.2. We may also consider the cases where $\Lambda_{1}<0, Y_{1}<0$ and/or $\Lambda_{2}<$ $0, Y_{2}<0$. These cases correspond to pseudo-Riemannian Ricci flows. Notice that in (2.8b), if $q_{i}<0$ and $b_{i}<0$, we can multiply the equation by -1 and obtain the equation for $\left|q_{i}\right|$ and $\left|b_{i}\right|$. Therefore, we can deduce the existence of ancient solutions from that of the positive case.

Another way to get a pseudo-Riemannian flow is to assume that the signs of $\tau$ and $\psi$ in equations (2.8a) and (2.8b) are both negative. By changing the sign of $\tau$ and $\psi$ and replacing $q_{i}$ by $\left|q_{i}\right|$ we obtain the equation for $|\tau|,|\psi|$ and $\left|q_{i}\right|$. Again we can deduce from the existence of ancient solutions for the positive case that there are immortal solutions of pseudo-Riemannian metrics on $\bar{P}$ which are negative definite on the fibres and positive definite on the base.

Note that there are many non-symmetric homogeneous negative QK manifolds, see e.g. Co96. As well, C. Lebrun showed that the moduli space of complete QK structures on $\mathbb{R}^{4 n}$ is infinite-dimensional [Le91. Finally, we mention the recent thesis of M. Dyckmanns Dy15] in which he constructed complete negative QK manifolds that are not locally homogeneous for all dimensions $4 n$.

3.2. Limiting behavior of the ancient solutions. Next we consider the longtime behavior of the solutions in Theorem 3.1. The arguments are analogous to those for the torus bundles in [LW16, §3.5], but because the fibres now have positive curvature, some of the conclusions are different.

Theorem 3.3. Let $Y(u)$ be one of the ancient solutions in Theorem [3.1(ia) with initial value $Y(0) \in \Omega_{1} \backslash\{\xi\}$. Then

(i) the forward limit $\lim _{u \rightarrow \infty} Y(u)=\eta$, and

(ii) there is exactly one solution whose backwards limit $\lim _{u \rightarrow-\infty} Y(u)=\xi$.

Proof. We use the notations in the proof of Proposition 2.6.

(i) Let $\omega_{Y}$ be the the $\omega$-limit set of the flow line $Y(u)$. Since

$$
\frac{d}{d u} E(Y(u))=-8 n_{1} q_{1}^{2} Y_{1}^{2} F_{1}(Y)-8 n_{2} q_{2}^{2} Y_{2}^{2} F_{2}(Y) \leq 0
$$

with equality if and only if $F_{1}(Y)=F_{2}(Y)=0$, it follows that $\omega_{Y}=\{\eta\}$ or $\{\xi\}$. Below we rule out the possibility that $\omega_{Y}=\{\xi\}$.

We claim that for any $Y(0) \in \Omega_{1} \backslash\{\xi\}$ there is an $i_{0}$ such that $Y_{i_{0}}(0)<\xi_{i_{0}}$. Assuming this claim, we compute that $\frac{d Y_{i_{0}}}{d u}(u)=-Y_{i_{0}}(u) F_{i_{0}}(u) \leq 0$ and so $\lim _{u \rightarrow \infty} Y_{i_{0}}(u)<$ $\xi_{i_{0}}$. Hence $\omega_{Y}=\{\eta\}$ must hold. 
We next prove the above claim by considering two cases. We rewrite the equations $F_{1}(Y)=F_{2}(Y)=0$ in the $\left(q_{1} Y_{1}, q_{2} Y_{2}\right)$-plane as loci of ellipses:

$$
\begin{aligned}
& \frac{\left(q_{1} Y_{1}-\frac{n_{1}+2}{4 n_{1}+6}\right)^{2}}{\left(\frac{n_{1}+1}{4 n_{1}+6}\right)^{2}}+\frac{\left(q_{2} Y_{2}\right)^{2}}{\frac{\left(n_{1}+1\right)^{2}}{\left(4 n_{1}+6\right) \cdot 4 n_{2}}}=1, \\
& \frac{\left(q_{1} Y_{1}\right)^{2}}{\frac{\left(n_{2}+1\right)^{2}}{\left(4 n_{2}+6\right) \cdot 4 n_{1}}}+\frac{\left(q_{2} Y_{2}-\frac{n_{2}+2}{4 n_{2}+6}\right)^{2}}{\left(\frac{n_{2}+1}{4 n_{2}+6}\right)^{2}}=1 .
\end{aligned}
$$

(ia) $n_{2} \geq n_{1} \geq 1$ and $\left(n_{1}, n_{2}\right) \neq(1,1)$. From (3.1a) we conclude that

$$
q_{2} \xi_{2} \leq \sqrt{\frac{\left(n_{1}+1\right)^{2}}{\left(4 n_{1}+6\right) \cdot 4 n_{2}}} .
$$

From (3.1b) we get

$$
q_{2} \xi_{2}=\frac{n_{2}+2 \pm \sqrt{\left(n_{2}+1\right)^{2}-\left(4 n_{2}+6\right) \cdot 4 n_{1} q_{1}^{2} \xi_{1}^{2}}}{4 n_{2}+6} .
$$

Since for $n_{2} \geq n_{1} \geq 1$ and $\left(n_{1}, n_{2}\right) \neq(1,1)$ one can check that

$$
\frac{n_{2}+2}{4 n_{2}+6}>\sqrt{\frac{\left(n_{1}+1\right)^{2}}{\left(4 n_{1}+6\right) \cdot 4 n_{2}}},
$$

it follows from (3.2) that we need to take negative sign in (3.3), and the square root in the equation cannot be zero. We have proven that $\left(q_{1} \xi_{1}, q_{2} \xi_{2}\right)$ lies in the lower-right quarter of the ellipse in the $\left(q_{1} Y_{1}, q_{2} Y_{2}\right)$-plane defined by $F_{2}(Y)=0$ and cannot be the right vertex of the ellipse. This implies that every point $\left(Y_{1}, Y_{2}\right) \in \Omega_{1} \backslash\{\xi\}$ satisfies $Y_{1}<\xi_{1}$.

(ib) $n_{2}=n_{1}=1$. Then by (3.1a) and (3.1b) we know that the set

$$
\left\{\left(q_{1} Y_{1}, q_{2} Y_{2}\right):\left(Y_{1}, Y_{2}\right) \in \Omega_{1}\right\}
$$

is a convex set in $\left(q_{1} Y_{1}, q_{2} Y_{2}\right)$-plane and is symmetric with respect to the diagonal. This implies that every point $\left(Y_{1}, Y_{2}\right) \in \Omega_{1} \backslash\{\xi\}$ satisfies either $Y_{1}<\xi_{1}$ or $Y_{2}<\xi_{2}$, otherwise $\xi$ will be an interior point of $\Omega_{1}$, which is not true. Now the claim is proved.

(ii) Let

$$
\begin{aligned}
& a_{i} \doteqdot 2\left(n_{i}+2\right) q_{i}-\left(4 n_{i}+6\right) \cdot 2 q_{i}^{2} \xi_{i}, \\
& b_{i} \doteqdot 8 n_{i} q_{i}^{2} \xi_{i}, \quad i=1,2 .
\end{aligned}
$$

We claim the following estimate

$$
\frac{b_{1}}{a_{1}+b_{1}}+\frac{b_{2}}{a_{2}+b_{2}}>1 \text {. }
$$

To verify the claim, note that $\nabla F_{1}(\xi)=\left(a_{1},-b_{2}\right)$ and $\nabla F_{2}(\xi)=\left(-b_{1}, a_{2}\right)$. From (ia) and (ib) above, there is a vector $\left(-\delta_{1},-\delta_{2}\right)$ with $\delta_{i}>0, i=1,2$, lying in the 
interior of the tangent cone $T_{\xi} \Omega_{1}$, i.e., there is a solution $\left(\lambda_{1}, \lambda_{2}\right)$ with $\lambda_{i}>0, i=1,2$, to the following equations,

$$
\begin{aligned}
a_{1} \lambda_{1}-b_{1} \lambda_{2} & =-\delta_{1}, \\
-b_{2} \lambda_{1}+a_{2} \lambda_{2} & =-\delta_{2}, \\
\lambda_{1}+\lambda_{2} & =1 .
\end{aligned}
$$

Note that it follows from $q_{i} \xi_{i}<\frac{1}{2}$ that

$$
a_{i}+b_{i}=2 q_{i}\left(\left(n_{i}+2\right)-6 q_{i} \xi_{i}\right)>0, \quad i=1,2 .
$$

We compute using (3.5a), (3.5b) and (3.5c) that

$$
\begin{aligned}
\frac{b_{1}}{a_{1}+b_{1}}+\frac{b_{2}}{a_{2}+b_{2}} & =\frac{\lambda_{1}\left(a_{1}+b_{1}\right)+\delta_{1}}{a_{1}+b_{1}}+\frac{\lambda_{2}\left(a_{2}+b_{2}\right)+\delta_{2}}{a_{2}+b_{2}} \\
& =1+\frac{\delta_{1}}{a_{1}+b_{1}}+\frac{\delta_{2}}{a_{2}+b_{2}}
\end{aligned}
$$

which gives the claim (3.4).

Next we claim that the eigenvalue $-E(\xi)+\rho_{1}(\xi)$ of $\mathcal{L}_{\xi}$, defined in Proposition 2.6(vii), is positive. To see this, we compute

$$
\begin{aligned}
0 & <\frac{b_{1}}{a_{1}+b_{1}}+\frac{b_{2}}{a_{2}+b_{2}}-1 \\
& =\frac{8 n_{1} q_{1}^{2} \xi_{1}^{2}}{E(\xi)-6 q_{1}^{2} \xi_{1}^{2}}+\frac{8 n_{2} q_{2}^{2} \xi_{2}^{2}}{E(\xi)-6 q_{2}^{2} \xi_{2}^{2}}-1 \\
& =\frac{G(\xi, E(\xi))}{\left(E(\xi)-6 q_{1}^{2} \xi_{1}^{2}\right) \cdot\left(E(\xi)-6 q_{2}^{2} \xi_{2}^{2}\right)},
\end{aligned}
$$

where for $\tilde{E} \in \mathbb{R}$

$$
\begin{aligned}
G(\xi, \tilde{E}) \doteqdot & -\tilde{E}^{2}+\left(\left(8 n_{1}+6\right) q_{1}^{2} \xi_{1}^{2}+\left(8 n_{2}+6\right) q_{2}^{2} \xi_{2}^{2}\right) \tilde{E} \\
& -\left(48 n_{1}+48 n_{2}+36\right) q_{1}^{2} q_{2}^{2} \xi_{1}^{2} \xi_{2}^{2}
\end{aligned}
$$

It is easy to check that $E(\xi)-6 q_{i}^{2} \xi_{i}^{2}>0, i=1,2$. Hence we conclude that $G(\xi, E(\xi))>0$.

Since $\rho_{1}(\xi)$ is an eigenvalue of the matrix $\alpha_{\xi}$ defined by (2.25), $\rho_{1}(\xi)$ satisfies $G\left(\xi, \rho_{1}(\xi)\right)=0$. Hence

$$
\begin{aligned}
0 & <G(\xi, E(\xi))-G\left(\xi, \rho_{1}(\xi)\right) \\
& =\left(-E(\xi)+\rho_{1}(\xi)\right) \cdot\left(E(\xi)+\rho_{1}(\xi)-\left(8 n_{1}+6\right) q_{1}^{2} \xi_{1}^{2}-\left(8 n_{2}+6\right) q_{2}^{2} \xi_{2}^{2}\right) .
\end{aligned}
$$

Note that $E(\xi)-6 q_{1}^{2} \xi_{1}^{2}>0$, and that by applying the Perron-Frobenius theory to the matrix $\alpha_{\xi}$ we have $\rho_{1}(\xi) \geq 8 n_{1} q_{1}^{2} \xi_{1}^{2}+\left(8 n_{2}+6\right) q_{2}^{2} \xi_{2}^{2}$ (see p.76 of [Ga59] for example). Hence the second factor in (3.6) is positive and so $-E(\xi)+\rho_{1}(\xi)>0$.

Now we can finish the proof of (ii) by using the Hartman-Grobman Theorem since the local unstable manifold at $\xi$ has dimension 1.

When the solution $Y(u)$ starts in $\Omega_{2}$, its longtime behavior is given by 
Theorem 3.4. Let $Y(u)$ be one of the ancient solutions in Theorem 3.1(ib) with initial value $Y(0) \in \Omega_{2} \backslash\left(\overline{0 v_{1}} \cup \overline{0 v_{2}} \cup\{\eta\}\right)$ where $\overline{0 v_{i}}$ denotes the line segment joining 0 and $v_{i}$. Then

(i) the forward limit $\lim _{u \rightarrow \infty} Y(u)=\eta$.

(ii) There is exactly one solution whose backwards limit $\lim _{u \rightarrow-\infty} Y(u)=v_{1}$. We denote the corresponding flow line by $\gamma_{1}$.

(iii) There is exactly one solution whose backwards limit $\lim _{u \rightarrow-\infty} Y(u)=v_{2}$. We denote the corresponding flow line by $\gamma_{2}$.

(iv) Let $\Omega_{2}^{*} \subset \Omega_{2}$ be the closed region bounded by $\overline{0 v_{1}}, \overline{0 v_{2}}, \gamma_{1}$ and $\gamma_{2} \cup\{\eta\}$. For each $Y(0)$ in the interior of $\Omega_{2}^{*}$ the solution $Y(u)$ has backwards limit $\lim _{u \rightarrow-\infty} Y(u)=0$.

Proof. We use the notations in the proof of Proposition 2.6.

(i) Let $\omega_{Y}$ be the $\omega$-limit set of the flow line $Y(u)$. Then

$$
\frac{d}{d u} E(Y(u))=-8 n_{1} q_{1}^{2} Y_{1}^{2} F_{1}(Y)-8 n_{2} q_{2}^{2} Y_{2}^{2} F_{2}(Y) \geq 0
$$

with equality if and only if $Y_{1} F_{1}(Y)=Y_{2} F_{2}(Y)=0$. As $\omega_{Y}$ is flow-invariant and connected, it follows that $\omega_{Y}=\{0\},\left\{v_{1}\right\},\left\{v_{2}\right\}$, or $\{\eta\}$. Below we rule out the possibilities that $\omega_{Y}=\{0\},\left\{v_{1}\right\}$, or $\left\{v_{2}\right\}$.

From (ia) and(ib) in the proof of Theorem 3.3 we conclude that $\left(q_{1} \eta_{1}, q_{2} \eta_{2}\right)$ lies in the right-lower quarter of the ellipse in $\left(q_{1} Y_{1}, q_{2} Y_{2}\right)$-plane defined by $F_{2}(Y)=0$ and cannot be the right vertex of the ellipse. Likewise, the point lies in the upper-left quarter of the ellipse $F_{1}(Y)=0$. These facts imply that that $Y_{i}(0)<\eta_{i}$ for any $Y(0) \in \Omega_{2} \backslash\{\eta\}$. But $\frac{d Y_{i}}{d u}(u)=-Y_{i}(u) F_{i}(u) \geq 0$, and the inequality is strict for at least one $i$ by the assumption on $Y(0)$. This implies that $\omega_{Y}=\{\eta\}$.

(ii) and (iii) Both follow from the Hartman-Grobman Theorem as in the proof of Theorem 3.3(ii).

(iv) Since the boundary of $\Omega_{2}^{*}$ consist of flow lines, by the uniqueness of solutions of ODEs, $Y(u)$ must stay in $\Omega_{2}^{*}$. From $\frac{d Y_{i}}{d u}(u)>0$ proved in (i), the backward limit must be one of $0, v_{1}$, and $v_{2}$. The last two limits are ruled out by the uniqueness in (ii) and (iii).

3.3. Asymptotic behavior of metric tensors of the ancient solutions. We begin with the following.

Theorem 3.5. Let $\left(\psi(\tau), b_{1}(\tau), b_{2}(\tau)\right)$ be one of the ancient solutions of the ODE system (2.8a) and (2.8b) given in Theorem 3.1(i). Then we have the following estimates and asymptotics.

(i) The domain for $\tau$ contains $[0, \infty)$. For any $\varepsilon>0$ small there is a $\tau_{\varepsilon}>0$ such that for any $\tau>\tau_{\varepsilon}$ and for $i=1,2$,

$$
\begin{aligned}
& (E(\eta)-\varepsilon)\left(\tau-\tau_{\varepsilon}\right) \leq \psi(\tau)-\psi\left(\tau_{\varepsilon}\right) \leq(E(\eta)+\varepsilon)\left(\tau-\tau_{\varepsilon}\right), \\
& -\varepsilon\left(\tau-\tau_{\varepsilon}\right) \leq b_{i}(\tau)-b_{i}\left(\tau_{\varepsilon}\right)-\left(2\left(n_{i}+2\right) q_{i}-6 q_{i}^{2} \eta_{i}\right)\left(\tau-\tau_{\varepsilon}\right) \leq \varepsilon\left(\tau-\tau_{\varepsilon}\right) .
\end{aligned}
$$

Furthermore, we have

$$
\lim _{\tau \rightarrow \infty} \frac{\psi(\tau)}{b_{i}(\tau)}=\eta_{i}, \quad \lim _{\tau \rightarrow \infty} \frac{\psi(\tau)}{\tau}=E(\eta)
$$


and so the rescaled metrics $\frac{1}{\tau} \bar{g}(\tau)$ converge in the Gromov-Hausdorff topology to a multiple of the Einstein metric corresponding to $\eta$ as $\tau$ approaches $\infty$.

(ii) For the solution in Theorem 3.3(ii) there is a finite $T_{1}>0$ such that when $u \rightarrow-\infty$ the corresponding $\tau \rightarrow-T_{1}^{+}$. We have

$$
\lim _{\tau \rightarrow-T_{1}^{+}} \psi(\tau)=0, \quad \lim _{\tau \rightarrow-T_{1}^{+}} b_{i}(\tau)=0, \quad \lim _{\tau \rightarrow-T_{1}^{+}} \frac{\psi(\tau)}{b_{i}(\tau)}=\xi_{i}, \quad \lim _{\tau \rightarrow-T_{1}^{+}} \frac{\psi(\tau)}{T_{1}+\tau}=E(\xi) .
$$

It follows that geometrically, as $\tau \rightarrow-T_{1}^{+}$, the $\mathbb{R P}^{3}$ fibre bundle $\bar{P}$, equipped with the metric $\bar{g}(\tau)$, collapses to a point. Furthermore, the rescaled metrics $\frac{1}{T_{1}+\tau} \bar{g}(\tau)$ converges to a multiple of the Einstein metric corresponding to $\xi$ as $\tau \rightarrow-T_{1}^{+}$.

Proof. (i) We first note that the range of $\tau(u)$ (as determined by (2.9)) contains $[0, \infty)$ for any of the solutions $Y(u)$ under consideration. The inequalities in (3.7) follow from $\frac{d \psi}{d \tau}=E(Y)$ and $\lim _{u \rightarrow \infty} Y(u)=\eta$ and those in (3.8) follow from (2.8b) and $\lim _{u \rightarrow \infty} Y(u)=\eta$. Note that $2\left(n_{i}+2\right) q_{i}-6 q_{i}^{2} \eta_{i}>0$, since we have $q_{i} \eta_{i}<\frac{1}{2}$ from the proof of Lemma 2.4(ii). The values of the limits follow from the convergence of $Y(u)$ and (2.8a).

(ii) The proof is similar to that of [LW16, Theorem 3.1.3(ii)]. We first show that $\lim _{u \rightarrow-\infty} \tau(u)=-T_{1}$ for some finite positive $T_{1}$. Since $\lim _{u \rightarrow-\infty} Y(u)=\xi$, we have

$$
\frac{d \psi}{d \tau}>\frac{1}{2}+n_{1} q_{1}^{2} \xi_{1}^{2}+n_{2} q_{2}^{2} \xi_{2}^{2}
$$

for $\tau$ corresponding to $u \in\left(-\infty, u_{*}\right]$. Here $u_{*}$ is a sufficiently negative number. Integrating this inequality over the $\tau$-interval corresponding to $\left[u, u_{*}\right]$ gives

$$
0<\tau\left(u_{*}\right)-\tau(u)<\psi\left(u_{*}\right)\left(\frac{1}{2}+n_{1} q_{1}^{2} \xi_{1}^{2}+n_{2} q_{2}^{2} \xi_{2}^{2}\right)^{-1} .
$$

This implies the existence of finite $T_{1}>0$.

Next one shows that $\lim _{\tau \rightarrow-T_{1}^{+}} \psi(\tau)=0$. The limit certainly exists as $\psi(\tau)$ is positive and increasing in $\tau$. If the limit is positive, we immediately get a contradiction to $\lim _{\tau \rightarrow-T_{1}^{+}} u(\tau)=\infty$ upon integrating $\frac{d u}{d \tau}=\frac{1}{\psi}$. We omit the remaining details as they are straight-forward.

When the solution $Y(u)$ starts from $\Omega_{2}$ the asymptotic behavior of the metric tensor is given by the next theorem.

Theorem 3.6. Let $\left(\psi(\tau), b_{1}(\tau), b_{2}(\tau)\right)$ be a solution of the ODE system (2.8a) and (2.8b).

(i) If it corresponds to the solution in Theorem $3.4\left(\right.$ ii) then there is a finite $T_{2}>0$ such that when $u \rightarrow-\infty$ the corresponding $\tau \rightarrow-T_{2}^{+}$. We in addition have

$$
\begin{aligned}
& \lim _{\tau \rightarrow-T_{2}^{+}} \psi(\tau)=0, \quad \lim _{\tau \rightarrow-T_{2}^{+}} b_{1}(\tau)=0, \quad \lim _{\tau \rightarrow-T_{2}^{+}} b_{2}(\tau)>0, \\
& \lim _{\tau \rightarrow-T_{2}^{+}} \frac{\psi(\tau)}{b_{1}(\tau)}=\frac{1}{\left(4 n_{1}+6\right) q_{1}}, \quad \lim _{\tau \rightarrow-T_{2}^{+}} \frac{\psi(\tau)}{b_{2}(\tau)}=0, \quad \lim _{\tau \rightarrow-T_{2}^{+}} \frac{\psi(\tau)}{T_{2}+\tau}=E\left(v_{1}\right) .
\end{aligned}
$$

Geometrically, as $\tau \rightarrow-T_{2}^{+}$, the $\mathbb{R P}^{3}$ fibre bundle $\bar{P}$, equipped with the metric $\bar{g}(\tau)$, collapses along the fibres and the first factor to some $Q K$ metric on the second factor 
$M_{2}$. The rescaled metrics $\frac{1}{T_{2}+\tau} \bar{g}(\tau)$ converge, as $\tau \rightarrow-T_{2}^{+}$, to product $P_{1} \times \mathbb{R}^{4 n_{2}}$ in the Gromov-Hausdorff topology, where $P_{1}$ is equipped with a multiple of the "squashed" Einstein metric (see the remark below) and $\mathbb{R}^{4 n_{2}}$ is equipped with the Euclidean metric. (ii) If it corresponds to the solution in Theorem 3.4(iii) then there is a finite $T_{3}>0$ such that when $u \rightarrow-\infty$ the corresponding $\tau \rightarrow-T_{3}^{+}$. We further have

$$
\begin{aligned}
& \lim _{\tau \rightarrow-T_{3}^{+}} \psi(\tau)=0, \quad \lim _{\tau \rightarrow-T_{3}^{+}} b_{1}(\tau)>0, \quad \lim _{\tau \rightarrow-T_{3}^{+}} b_{2}(\tau)=0, \\
& \lim _{\tau \rightarrow-T_{3}^{+}} \frac{\psi(\tau)}{b_{1}(\tau)}=0, \quad \lim _{\tau \rightarrow-T_{3}^{+}} \frac{\psi(\tau)}{b_{2}(\tau)}=\frac{1}{\left(4 n_{2}+6\right) q_{2}}, \quad \lim _{\tau \rightarrow-T_{3}^{+}} \frac{\psi(\tau)}{T_{3}+\tau}=E\left(v_{2}\right) .
\end{aligned}
$$

Geometrically, as $\tau \rightarrow-T_{3}^{+}$, the bundle $\bar{P}$, equipped with the metric $\bar{g}(\tau)$, collapses along the fibres and the second factor to some $Q K$ metric on the first factor $M_{1}$. The rescaled metrics $\frac{1}{T_{3}+\tau} \bar{g}(\tau)$ converge, as $\tau \rightarrow-T_{3}^{+}$, to product $\mathbb{R}^{4 n_{1}} \times P_{2}$ in the Gromov-Hausdorff topology, where $\mathbb{R}^{4 n_{1}}$ is equipped with the Euclidean metric and $P_{2}$ is equipped with a multiple of the "squashed" Einstein metric.

(iii) If it corresponds to a solution in Theorem 3.4 (iv) then there is a finite $T_{4}>0$ such that when $u \rightarrow-\infty$ the corresponding $\tau \rightarrow-T_{4}^{+}$. We also have

$$
\lim _{\tau \rightarrow-T_{4}^{+}} \psi(\tau)=0, \quad \lim _{\tau \rightarrow-T_{4}^{+}} b_{i}(\tau)>0, \quad \lim _{\tau \rightarrow-T_{4}^{+}} \frac{\psi(\tau)}{b_{i}(\tau)}=0, \quad \lim _{\tau \rightarrow-T_{4}^{+}} \frac{\psi(\tau)}{T_{4}+\tau}=\frac{1}{2} .
$$

Geometrically, as $\tau \rightarrow-T_{4}^{+}$, the bundle $\bar{P}$, equipped with the metric $\bar{g}(\tau)$, collapses along the fibres to the product metric

$$
\lim _{\tau \rightarrow-T_{4}^{+}} b_{1}(\tau) \cdot g_{1}+\lim _{\tau \rightarrow-T_{4}^{+}} b_{2}(\tau) \cdot g_{2}
$$

on $M_{1} \times M_{2}$. By choosing different initial data the ratio $\frac{\lim _{\tau \rightarrow-T_{4}^{+}} b_{2}(\tau)}{\lim _{\tau \rightarrow-T_{4}^{+}} b_{1}(\tau)}$ can be any positive number. The rescaled metrics $\frac{1}{T_{4}+\tau} \bar{g}(\tau)$ converge, as $\tau \rightarrow-T_{4}^{+}$, to product $\mathbb{R P}^{3} \times \mathbb{R}^{4\left(n_{1}+n_{2}\right)}$ in the Gromov-Hausdorff topology, where $\mathbb{R P}^{3}$ is equipped with the metric with constant sectional curvature $1 / 4$ and $\mathbb{R}^{4\left(n_{1}+n_{2}\right)}$ is equipped with the Euclidean metric.

Proof. (i) The proof is similar to that of Theorem 3.5(ii) except for the assertion that $\lim _{\tau \rightarrow-T_{2}^{+}} b_{2}(\tau)>0$. To prove this, we first give an estimate of $T_{2}$. Using again (2.10a) and the monotonicity of $E(Y(u))$, we have $E(0) \leq E(Y(u))=\frac{d \ln \psi}{d u} \leq E(\eta)$. Integrating this inequality over the interval $[u, 0]$ we get

$$
\psi(0) e^{E(\eta) u} \leq \psi(\tau(u))=\frac{d \tau}{d u} \leq \psi(0) e^{E(0) u},
$$

which in particular gives $\lim _{u \rightarrow-\infty} \psi(\tau(u))=0$. Integrating again, we get for $u<0$

$$
\frac{\psi(0)}{E(\eta)}\left(1-e^{E(\eta) u}\right) \leq-\tau(u) \leq \frac{\psi(0)}{E(0)}\left(1-e^{E(0) u}\right) .
$$

Hence by letting $u \rightarrow-\infty$ we get

$$
\frac{\psi(0)}{E(\eta)} \leq T_{2} \leq \frac{\psi(0)}{E(0)}=2 \psi(0)
$$


By integrating (2.8b) we get that $b_{2}(\tau) \geq b_{2}(0)+2 q_{2}\left(n_{2}+2\right) \tau$ for $\tau<0$. By using (3.9) we have

$$
\lim _{\tau \rightarrow-T_{2}^{+}} b_{2}(\tau) \geq b_{2}(0)-4 q_{2}\left(n_{2}+2\right) \psi(0)=b_{2}(0)\left(1-4 q_{2}\left(n_{2}+2\right) Y_{2}(0)\right)>0 .
$$

To get the last inequality, we may need to shift the initial time so that $q_{2} Y_{2}(0)<$ $\left(4\left(n_{2}+2\right)\right)^{-1}$, which is always possible. The remaining limits follow from the above and L'Hopital's rule.

(ii) Since the proof is similar to that of (i) above, we omit it.

(iii) Except for the last limit $\frac{\lim _{\tau \rightarrow-T_{4}^{+}} b_{2}(\tau)}{\lim _{\tau \rightarrow-T_{4}^{+}} b_{1}(\tau)}$, the rest of the proof is similar to that of (i) above and so we omit it. To deduce the limit, we use (2.23) together with Sternberg's Theorem ([St57, Theorem 4]) on the $C^{k}$-conjugacy of solutions near a fixed point of a nonlinear ODE system satisfying non-resonance conditions to solutions of its linearization. It follows that there is a diffeomorphism $W$ from a neighborhood of the origin in $\left(Y_{1}, Y_{2}\right)$-space to some neighborhood of $0 \in \mathbb{R}^{2}$ such that the flow lines $Y(u)$ satisfy

$$
Y(u)=\left(W^{-1} \circ\left(k_{1} e^{u / 2}, k_{2} e^{u / 2}\right) \circ W\right)(Y(0))
$$

where $u$ is sufficiently close to $-\infty$. Taking the $u$-derivative of this equation and letting $u$ tend to $-\infty$ we see that the tangent map $W_{*}^{-1}$ maps directions at 0 with slope $k_{2} / k_{1}$ to directions at 0 in $Y$-plane. In particular, as $\overline{0 v_{1}}$ and $\overline{0 v_{2}}$ are themselves flow lines, it follows that there are flow lines $Y(u)$ emanating from 0 which lie in between $\overline{0 v_{1}}$ and $\overline{0 v_{2}}$ with any positive limiting slope.

Remark 3.7. Here we clarify the term "squashed" Einstein metric for readers not familiar with Einstein metrics on the principal $\mathrm{SO}(3)$ bundle $P$ over a compact QK manifold. We will use the notation in \$2.1. It is well-known (see [Be87, Proposition 14.85]) that $P$ admits two Einstein metrics of connection type. Indeed, they can be found by solving the quadratic equation resulting from (2.12) by dropping the terms with index 2. The two solutions are then given by $Y_{1}=\frac{1}{2 q_{1}}$ and $Y_{1}=\frac{1}{\left(4 n_{1}+6\right) q_{1}}$. The latter is what we refer to as the "squashed" Einstein metric. The terminology comes from the case when the QK manifold is quaternionic projective space $\mathbb{H P}^{n}$ and $P$ is $S^{4 n+3}$. The "squashed" Einstein metric is the Jensen metric, obtained from the constant curvature metric by uniformly shrinking the Hopf fibres. It is of interest to physicists since the dimension of the space of Killing spinors is 1 . There are also pseudo-Riemannian analogues of these Einstein metrics (see [Be87, Remark 14.86b]), which appear as limits in our construction of pseudo-Riemannian flows.

Theorem 3.8. Let $\left(\psi(\tau), b_{1}(\tau), b_{2}(\tau)\right)$ be one of the ancient solutions of the ODE system (2.8a) and (2.8b) in Theorem 3.1 (ii). Then the domain for $\tau$ contains $[0, \infty)$ and for any small $\varepsilon>0$ there is a $\tau_{\varepsilon}>0$ such that for $\tau \geq \tau_{\varepsilon}$ and for $i=1,2$,

$$
\begin{aligned}
& (E(\xi)-\varepsilon)\left(\tau-\tau_{\varepsilon}\right) \leq \psi(\tau)-\psi\left(\tau_{\varepsilon}\right) \leq(E(\xi)+\varepsilon)\left(\tau-\tau_{\varepsilon}\right), \\
& \left(2 n_{i}+1\right) q_{i}\left(\tau-\tau_{\varepsilon}\right) \leq b_{i}(\tau)-b_{i}\left(\tau_{\varepsilon}\right) \leq 2\left(n_{i}+2\right) q_{i}\left(\tau-\tau_{\varepsilon}\right) .
\end{aligned}
$$

Furthermore, we have

$$
\lim _{\tau \rightarrow \infty} \frac{\psi(\tau)}{b_{i}(\tau)}=\xi_{i}, \quad \lim _{\tau \rightarrow \infty} \frac{\psi(\tau)}{\tau}=E(\xi),
$$


and so the rescaled metrics $\frac{1}{\tau} \bar{g}(\tau)$ converge in the Gromov-Hausdorff topology to a multiple of the Einstein metric corresponding to $\xi$ as $\tau$ approaches $\infty$.

Proof. Inequalities (3.10) follows from $\frac{d \psi}{d \tau}=E(Y)$ and $\lim _{u \rightarrow \infty} Y(u)=\xi$. Since $q_{i} \xi_{i}<$ $\frac{1}{2}$, we may choose $\tau_{\varepsilon}$ so that by using (2.8b) we have $\left(2 n_{i}+1\right) q_{i} \leq \frac{d b_{i}}{d \tau} \leq 2\left(n_{i}+2\right) q_{i}$ for $\tau \geq \tau_{\varepsilon}$. Then the inequality (3.11) follows. The remaining convergences follow easily.

With the asymptotics given in Theorems 3.5, 3.6 and 3.8, one easily deduces the following

Corollary 3.9. (i) Each of the ancient solutions $\bar{g}(\tau), \tau \in[0, \infty)$, in Theorem 3.1, is $\kappa$-noncollapsed at all scales for some $\kappa>0$.

(ii) Each of the solutions $\bar{g}(\tau)$ on $\tau \in\left(-T_{i}, 0\right], 1 \leq i \leq 4$ in Theorems [3.5(ii) and 3.6. (i)-(iii), is $\kappa$-noncollapsed at all scales for some $\kappa>0$.

\section{4. $C^{0}$ Cheeger-Gromov convergence implies $C^{\infty}$ Cheeger-Gromov convergence and Type I solutions of the Ricci flow}

Although we can compute the curvature tensor of the ancient solutions we found in Theorem [3.1 as we did for the torus bundle case in [LW16] and conclude that these solutions are of type I, in this section we will instead prove the solutions are of type I by an indirect method. This relies on a result relating $C^{0}$ and $C^{\infty}$ convergence of Ricci flow solutions under suitable hypotheses. We believe that this result may of be independent interest and have other applications.

Recall the following definition of convergence in pointed $C^{k}$ Cheeger-Gromov topology, $0 \leq k \leq \infty$.

Definition 4.1. Let $I$ be a fixed interval. A sequence $\left\{\left(M_{k}^{n}, g_{k}(t), p_{k}\right)\right\}, t \in I$, of complete pointed solutions of the Ricci flow converges in the $C^{k}$ (pointed) CheegerGromov topology to a complete pointed solution $\left(M_{\infty}^{n}, g_{\infty}(t), p_{\infty}\right), t \in I$, of the Ricci flow if there exist

(i) an exhaustion $\left\{U_{k}\right\}_{k \in \mathbb{N}}$ of $M_{\infty}$ by open sets with $p_{\infty} \in U_{k}$ for each $k$, and

(ii) a sequence of diffeomorphisms $\Phi_{k}: U_{k} \rightarrow \Phi_{k}\left(U_{k}\right) \subset M_{k}$ with $\Phi_{k}\left(p_{\infty}\right)=p_{k}$

such that $\left(U_{k}, \Phi_{k}^{*}\left(\left.g_{k}(t)\right|_{\Phi_{k}\left(U_{k}\right)}\right)\right)$ converges in the $C^{k}$ topology to $\left(M_{\infty}, g_{\infty}(t)\right)$ uniformly on any compact subset in $M_{\infty} \times I$.

We have the following strengthening of $C^{0}$ Cheeger-Gromov convergence of the Ricci flow.

Theorem 4.2. Let $\left\{\left(M_{k}^{n}, g_{k}(t), p_{k}\right)\right\}, t \in[0,1]$, be a sequence of complete pointed solutions of the Ricci flow with bounded curvature for each $k$. We assume that

(a) the scalar curvature $R_{g_{k}}(x, t) \geq-R_{*}$ on $M_{k} \times[0,1]$ for some constant $R_{*}>0$,

(b) the sequence $\left\{\left(M_{k}, g_{k}(t), p_{k}\right)\right\}, t \in[0,1]$, converges in the $C^{0}$ Cheeger-Gromov topology to complete pointed solution $\left(M_{\infty}^{n}, g_{\infty}(t), p_{\infty}\right), t \in[0,1]$, of the Ricci flow.

Then the sequence $\left\{\left(M_{k}, g_{k}(t), p_{k}\right)\right\}, t \in(0,1]$, subconverges to $\left(M_{\infty}, g_{\infty}(t), p_{\infty}\right), t \in$ $(0,1]$, in $C^{\infty}$ Cheeger-Gromov topology. 
Proof. Fix $\alpha=1$ and let $\delta$ and $\epsilon_{0}<1$ be the two positive constants in Perelman's pseudolocality theorem ([Pe02, Theorem 10.1]). We claim that for any compact subset $K \subset M_{\infty}$ there is a $r_{*} \in\left(0, \min \left\{1 / 2, R_{*}^{-1 / 2}\right\}\right)$ such that for any $x_{*} \in K$ and any $t \in[0,1]$ the ball $B_{g_{\infty}(t)}\left(x_{*}, 2 r_{*}\right)$ is $\delta / 2$-almost isoperimetrically Euclidean in the sense

$$
\left(\operatorname{Area}_{g_{\infty}(t)}(\partial \Omega)\right)^{n} \geq\left(1-\frac{\delta}{2}\right) c_{n}\left(\operatorname{Vol}_{g_{\infty}(t)}(\Omega)\right)^{n-1}
$$

for any regular domain $\Omega \subset B_{g_{\infty}(t)}\left(x_{*}, 2 r_{*}\right)$, where $c_{n}=n^{n} \omega_{n}$ is the Euclidean isoperimetric constant.

Proof of the claim. Because every Riemannian metric is infinitesimally a Euclidean metric and the isoperimetric property is stable for nearly equivalent metrics, given any $(\tilde{x}, \tilde{t}) \in M_{\infty} \times[0,1]$ there are $\tilde{r}>0$ and $\tilde{\eta}>0$ sufficiently small such that

$$
\left(\operatorname{Area}_{g_{\infty}(t)}(\partial \Omega)\right)^{n} \geq\left(1-\frac{\delta}{2}\right) c_{n}\left(\operatorname{Vol}_{g_{\infty}(t)}(\Omega)\right)^{n-1}
$$

for any regular domain $\Omega \subset B_{g_{\infty}(t)}(\tilde{x}, 4 \tilde{r})$ and any $t \in I_{\tilde{t}, \tilde{\eta}}$. We define interval

$$
I_{\tilde{t}, \tilde{\eta}} \doteqdot \begin{cases}(\max \{\tilde{t}-\tilde{\eta}, 0\}, \min \{\tilde{t}+\tilde{\eta}, 1\}) & \text { if } \tilde{t}-\tilde{\eta}>0 \text { and } \tilde{t}+\tilde{\eta}<1 \\ {[0, \min \{\tilde{t}+\tilde{\eta}, 1\})} & \text { if } \tilde{t}-\tilde{\eta} \leq 0 \text { and } \tilde{t}+\tilde{\eta}<1 \\ (\max \{\tilde{t}-\tilde{\eta}, 0\}, 1] & \text { if } \tilde{t}-\tilde{\eta}>0 \text { and } \tilde{t}+\tilde{\eta} \geq 1 \\ {[0,1]} & \text { if } \tilde{t}-\tilde{\eta} \leq 0 \text { and } \tilde{t}+\tilde{\eta} \geq 1\end{cases}
$$

Note that

$$
\left\{\cup_{t \in I_{\tilde{t}, \tilde{\eta}}} B_{g_{\infty}(t)}(\tilde{x}, 2 \tilde{r}) \times\{t\},(\tilde{x}, \tilde{t}) \in M_{\infty} \times[0,1]\right\}
$$

is an open cover of $M_{\infty} \times[0,1]$. Hence for any compact subset $K \subset M_{\infty}$, we can extract a finite subcovering $\left\{\cup_{t \in I_{\tilde{t}_{i}, \tilde{\eta}_{i}}} B_{g_{\infty}(t)}\left(\tilde{x}_{i}, 2 \tilde{r}_{i}\right) \times\{t\}, i=1,2, \cdots, i_{K}\right\}$ of $K \times[0,1]$. We choose $r_{*}$ to be any positive number less than both $\min _{i=1,2, \cdots, i_{K}} \tilde{r}_{i}$ and $1 / 2$.

Now we verify the conclusion of the claim. Given any $\left(x_{*}, t_{*}\right) \in K \times[0,1]$ there is an $i$ such that $\left(x_{*}, t_{*}\right) \in \cup_{t \in I_{\tilde{t}_{i}, \tilde{\eta}_{i}}} B_{g_{\infty}(t)}\left(\tilde{x}_{i}, 2 \tilde{r}_{i}\right) \times\{t\}$. Then given any regular domain $\Omega \subset B_{g_{\infty}\left(t_{*}\right)}\left(x_{*}, 2 r_{*}\right)$ we have $\Omega \subset B_{g_{\infty}\left(t_{*}\right)}\left(\tilde{x}_{i}, 4 \tilde{r}_{i}\right)$ where $t_{*} \in I_{\tilde{t}_{i}, \tilde{\eta}_{i}}$. Hence by the choice of $\tilde{x}_{i}, \tilde{t}_{i}, \tilde{r}_{i}$, and $\tilde{\eta}_{i}$ in (4.2) we know that inequality (4.1) holds for $\Omega$ and the claim holds.

Since limit manifold $M_{\infty}$ may be noncompact, below we will use balls centered at $p_{\infty}$ with larger and larger radius to provide the exhaustion in Definition 4.1. Fix any $D>0$. By assumption (b) the metrics $\Phi_{k}^{*} g_{k}(t)$ converges in $C^{0}$ topology to $g_{\infty}(t)$ uniformly on $\cup_{t \in[0,1]} B_{g_{\infty}(t)}\left(p_{\infty}, D+2\right) \times\{t\}$. Using this fact, the claim, and the property that the isoperimetric property is stable for nearly equivalent metrics, we conclude that there is an $r_{*} \in\left(0, \min \left\{1 / 2, R_{*}^{-1 / 2}\right\}\right)$ which is independent of $k$ and $k_{0}$ such that for any $k \geq k_{0}, t \in[0,1]$, and $x_{0} \in B_{g_{k}(t)}\left(p_{k}, D\right)$ we have

$$
\left(\operatorname{Area}_{g_{k}(t)}(\partial \Omega)\right)^{n} \geq(1-\delta) c_{n}\left(\operatorname{Vol}_{g_{k}(t)}(\Omega)\right)^{n-1}
$$

for any regular domain $\Omega \subset B_{g_{k}(t)}\left(x_{0}, r_{*}\right)$.

By (4.3),$r_{*} \leq R_{*}^{-1 / 2}$, and by the assumption (a) we may apply Perelman's pseudolocality theorem to the Ricci flow $\left(M_{k},\left.g_{k}(t)\right|_{t \in\left[t_{*}, t_{*}+\left(\epsilon_{0} r_{*}\right)^{2}\right]}\right)$ with $k \geq k_{0}$ and $t_{*} \in$ 
$\left[0,1-\left(\epsilon_{0} r_{*}\right)^{2}\right]$. We then obtain the interior curvature estimate

$$
\left|\operatorname{Rm}_{g_{k}}\right|(x, t) \leq \frac{1}{t-t_{*}}+\frac{1}{\left(\epsilon_{0} r_{*}\right)^{2}}
$$

where $d_{g_{k}(t)}\left(x, x_{0}\right)<\epsilon_{0} r_{*}, x_{0} \in B_{g_{k}(t)}\left(p_{k}, D\right)$, and $t \in\left(t_{*}, t_{*}+\left(\epsilon_{0} r_{*}\right)^{2}\right]$.

Given any $D>0$ and $\bar{t}>0$, by applying inequality (4.4) for some $t_{*} \in[\max \{0, \bar{t}-$ $\left.\left.\left(\epsilon_{0} r_{*}\right)^{2}\right\}, 1-\left(\epsilon_{0} r_{*}\right)^{2}\right]$ we get the uniform curvature bound

$$
\left|\mathrm{Rm}_{g_{k}}\right|(x, t) \leq \frac{1}{\bar{t}}+\frac{2}{\left(\epsilon_{0} r_{*}\right)^{2}}
$$

for $k \geq k_{0}, x \in B_{g_{k}(t)}\left(p_{k}, D\right)$, and $t \in[\bar{t}, 1]$.

Next we are going to prove that the sequence of injectivity radii $\operatorname{inj}_{g_{k}(1 / 2)}\left(p_{k}\right)$ have an uniform lower bound independent of $k$. By the assumption (b) we know that

$$
\lim _{k \rightarrow \infty} \operatorname{Vol}_{g_{k}(1 / 2)}\left(B_{g_{k}(1 / 2)}\left(p_{k}, 1\right)\right)=\operatorname{Vol}_{g_{\infty}(1 / 2)}\left(B_{g_{\infty}(1 / 2)}\left(p_{\infty}, 1\right)\right) .
$$

By (4.5) there is a constant $K_{0}$ such that the curvature $\left|\operatorname{Rm}_{g_{k}}\right|(x, 1 / 2) \leq K_{0}$ for any $x \in B_{g_{k}(1 / 2)}\left(p_{k}, 1\right)$ and $k \geq k_{0}$. Hence by Lemma 4.3 below there is a constant $\iota_{0}>0$ independent of $k$ such that $\operatorname{inj}_{g_{k}(1 / 2)}\left(p_{k}\right) \geq \iota_{0}$.

Now we state a local version of a theorem of Cheeger, Gromov, and Taylor (see CGT82, Theorem 4.7]).

Lemma 4.3. For any positive constants $r_{0}, k_{0}, v_{0}$, and dimension $n$, there exists a constant $\iota_{0}>0$ depending only on $r_{0}, k_{0}, v_{0}, n$ such that if for some precompact ball $B\left(p, r_{0}\right)$ in a Riemannian manifold $\left(M^{n}, g\right)$ the sectional curvature $|\operatorname{sect}(x)| \leq k_{0}$ for all $x \in B\left(p, r_{0}\right)$ and the volume $\operatorname{Vol}\left(B\left(p, r_{0}\right)\right) \geq v_{0}$, then the injectivity radius $\operatorname{inj}(p) \geq \iota_{0}$.

Proof. It is well known that $\operatorname{inj}(p)=\min \left\{\right.$ conjugate radius at $\left.p, \frac{1}{2} \ell(p)\right\}$, where $\ell(p)$ denotes the length of the shortest (nontrivial) closed geodesic starting and ending at $p$. The conjugate radius at $p$ is bounded from below by a constant $\iota_{1} \doteqdot \min \left\{r_{0}, \frac{\pi}{\sqrt{k_{0}}}\right\}$. It follows from [CGT82, Theorem 4.3] that $\ell(p)$ is bounded from below by a constant $\iota_{2}>0$ depending only on $r_{0}, k_{0}, v_{0}, n$. The theorem now is proved.

With the curvature bound (4.5) and the injectivity radius lower bound, we can now apply Hamilton's local compactness theorem ([Ha93, Theorem 16.1]) to sequence $\left\{\left(M_{k}, g_{k}(t), p_{k}\right)\right\}, t \in(0,1]$, with base time $t=1 / 2$ to conclude its subconvergence to $\left(\tilde{M}_{\infty}^{n}, \tilde{g}_{\infty}(t), \tilde{p}_{\infty}\right), t \in(0,1]$, in $C^{\infty}$ Cheeger-Gromov topology. By the uniqueness of the $C^{0}$ Cheeger-Gromov limit we know that $\left(\tilde{M}_{\infty}, \tilde{g}_{\infty}(t), \tilde{p}_{\infty}\right)$ is isometric to $\left(M_{\infty}, g_{\infty}(t), p_{\infty}\right)$ for each $t \in(0,1]$.

The following corollary shows that the ancient solutions we constructed in this article are of type I as time approaches to $-\infty$.

Corollary 4.4. Let $\bar{g}(\tau) \doteqdot \bar{g}_{\vec{a}(\tau), \vec{b}(\tau)}, \tau \in[0, \infty)$, be an ancient solution of the backwards Ricci flow on the compact fibre bundles $\bar{P}$ as defined in \$2.2. Assume that limits $\lim _{\tau \rightarrow \infty} \frac{a_{i}(\tau)}{\tau} \doteqdot \hat{a}_{i} \neq 0$ and $\lim _{\tau \rightarrow \infty} \frac{b_{i}(\tau)}{\tau} \doteqdot \hat{b}_{i} \neq 0$ for $i=1, \cdots, m$. Then the solution $\bar{g}(\tau)$ is of type $I$ when $\tau \rightarrow \infty$. 
Proof. It suffices to show that for any $\tau_{k} \rightarrow \infty$ there is a constant $C$ independent of $k$ such that $\sup _{p \in \bar{P}} \tau_{k}\left|\operatorname{Rm}_{\bar{g}\left(\tau_{k}\right)}(p)\right| \leq C$ for some subsequence of $\tau_{k}$. Note that $\left(\bar{P}, \bar{g}_{\overrightarrow{\hat{a}}, \vec{b}}\right)$ is a compact Riemannian manifold where $\overrightarrow{\hat{a}}=\left(\hat{a}_{1}, \cdots, \hat{a}_{m}\right)$ and $\overrightarrow{\hat{b}}=\left(\hat{b}_{1}, \cdots, \hat{b}_{m}\right)$.

Define the sequence of Ricci flow solutions $g_{k}(t)=\left(\tau_{k}\right)^{-1} \bar{g}\left(\frac{3}{2} \tau_{k}-\tau_{k} t\right), t \in[0,1]$. Since

$$
\lim _{k \rightarrow \infty} \frac{a_{i}\left(\frac{3}{2} \tau_{k}-\tau_{k} t\right)}{\tau_{k}}=\left(\frac{3}{2}-t\right) \hat{a}_{i}, \quad \lim _{k \rightarrow \infty} \frac{b_{i}\left(\frac{3}{2} \tau_{k}-\tau_{k} t\right)}{\tau_{k}}=\left(\frac{3}{2}-t\right) \hat{b}_{i},
$$

the sequence of solutions $\left\{g_{k}(t)\right\}$ satisfies the assumptions of Theorem 4.2 and it follows that $g_{k}\left(\frac{1}{2}\right)=\left(\tau_{k}\right)^{-1} \bar{g}\left(\tau_{k}\right)$ subconverges in $C^{\infty}$ Cheeger-Gromov topology to the compact Riemannian manifold $\left(\bar{P}, \bar{g}_{\overrightarrow{\hat{a}}, \vec{b}}\right)$. Since $\tau_{k}\left|\operatorname{Rm}_{\bar{g}\left(\tau_{k}\right)}\right|(p)=\left|\operatorname{Rm}_{g_{k}\left(\frac{1}{2}\right)}\right|(p)$ for any $p \in M$, we have the subconvergence of $\sup _{p \in \bar{P}} \tau_{k}\left|\operatorname{Rm}_{\bar{g}\left(\tau_{k}\right)}\right|(p)$ to $\sup _{p \in \bar{P}}\left|\operatorname{Rm}_{\bar{g}_{\vec{a}, \vec{b}}}\right|(p)$. Hence the subsequence of $\left\{\tau_{k}\left|\operatorname{Rm}_{\bar{g}\left(\tau_{k}\right)}\right|(p)\right\}$ is bounded.

Theorem 4.5. The ancient solutions $\bar{g}_{\vec{a}(\tau), \vec{b}(\tau)}$ of the Ricci flow on $\bar{P}$ with $m=2$ in Theorem 3.1 are of Type I when $\tau \rightarrow \infty$. The Ricci curvature of these solutions are positive definite for large enough $\tau$. The Ricci curvature of those solutions in Theorem 3.1 ( ia) and (ib) are positive for each $\tau$.

Proof. The first assertion follows from Corollary 4.4 directly.

For the second assertion, the formulas (2.1) and (2.6) imply that the Ricci tensors are positive definite provided that $Y_{i}<\frac{n_{i}+2}{6 q_{i}}$ for $i=1$, 2. Since $q_{i} \eta_{i}<q_{i} \xi_{i}<\frac{1}{2}$, we conclude that any solution $Y(u)$ in Theorem 3.1 satisfies the condition above when $u$ is large enough.

For the third assertion, in fact in the regions $\Omega_{i}, i=1,2$, we actually have $Y_{i}<$ $\frac{1}{2 q_{i}}$.

\section{Examples of ancient solutions with $m \geq 3$}

In this section we shall consider a special case of the Riemannian fibre bundles $\left(\bar{P}, \bar{g}_{\vec{a}, \vec{b}}\right)$ in $\S 2$ with $m \geq 3$ in order to exhibit continuous families of non-collapsed ancient solutions on even-dimensional non-Kähler manifolds. For the special case we assume that the QK manifolds $\left(M_{i}^{4 n_{i}}, g_{i}\right)$ are positive and have the same dimension, i.e., $n_{1}=\cdots=n_{m} \doteqdot d$. For convenience we shall assume that the constants $\Lambda_{i}=$ $n_{i}+2=d+2$ for all $i$, so that the constants $q_{i}=1$.

5.1. Properties of Einstein metrics on $\bar{P}$ with $m \geq 3$. Recall that for a solution $\bar{g}_{\vec{a}(\tau), \vec{b}(\tau)}=\bar{g}(\tau)$ of the backwards Ricci flow equations (2.4) and (2.1) we had set $\hat{a} \doteqdot \sum_{k=1}^{m} a_{k}$. We now let $X_{k} \doteqdot \frac{a_{k}}{\hat{a}}$ and $Y_{k} \doteqdot \frac{\hat{a}}{b_{k}}$, so that we have the constraint $\sum_{k=1}^{m} X_{k}=1$ for the variables $X_{k}$. Let $E(X, Y) \stackrel{m}{\doteqdot}+1+\sum_{k=1}^{m} 4 d X_{k}^{2} Y_{k}^{2}$, so that by (2.5) we have $\frac{d \hat{a}}{d \tau}=E(X, Y)$. Analogous to the $m=2$ case we define a new independent variable $u$ by $u=\int_{0}^{\tau} \frac{1}{\hat{a}(\zeta)} d \zeta$. Then it follows from (2.4) and (2.1) that

$$
\begin{aligned}
& \frac{d X_{k}}{d u}=\frac{1}{2}+X_{k}+4 d X_{k}^{2} Y_{k}^{2}-X_{k} E(X, Y), \\
& \frac{d Y_{k}}{d u}=-Y_{k}\left(2(d+2) Y_{k}-6 X_{k}\left(1-X_{k}\right) Y_{k}^{2}-E(X, Y)\right) .
\end{aligned}
$$


It is easy to check that condition $\sum_{k=1}^{m} X_{k}=1$ is preserved by (5.1a). Since the metrics we shall construct must satisfy this constraint, we will only consider solutions of the above system which satisfy $\sum_{k=1}^{m} X_{k}=1$ as well as the positivity conditions $X_{k}>0$ and $Y_{k}>0$. Given a solution of (5.1a) and (5.1b) we can find $\hat{a}(u)$ from $\frac{d \ln \hat{a}}{d u}=E(X, Y)$ first, picking up an integration constant in the process. Then we find $\tau(u)$ from $\frac{d \tau}{d u}=\hat{a}(u)$, and finally recover $a_{k}(\tau)$ and $b_{k}(\tau)$ from $X_{k}$ and $Y_{k}$. Hence we get solutions of the backwards Ricci flow depending on the integration constant. Note that the net effect of the integration constant is a parabolic rescaling of the solutions of the backwards Ricci flow. Hence given a solution of (5.1a) and (5.1b) we get only one solution $\bar{g}(\tau)$ of the backwards Ricci flow (modulo time translation and parabolic rescaling).

One can easily determine all the fixed points of the ODE system (5.1a) and (5.1b). Here we will examine those fixed points with all $Y_{k} \neq 0$. This leads to the equations

$$
\begin{aligned}
& \frac{1}{2}+X_{k}+4 d X_{k}^{2} Y_{k}^{2}-X_{k} E(X, Y)=0, \\
& 2(d+2) Y_{k}-6 X_{k}\left(1-X_{k}\right) Y_{k}^{2}-E(X, Y)=0 .
\end{aligned}
$$

Let $E(X, Y) \doteqdot 2 \Lambda \hat{a}$. Then after some simple calculation we obtain

$$
\begin{aligned}
& \frac{1}{4}+\frac{a_{k}}{2 \hat{a}}+2 d \frac{a_{k}^{2}}{b_{k}^{2}}=\Lambda a_{k}, \\
& d+2-3\left(1-\frac{a_{k}}{\hat{a}}\right) \frac{a_{k}}{b_{k}}=\Lambda b_{k} .
\end{aligned}
$$

By the Ricci curvature formulas in $\$ 2.2$, these are exactly the Einstein condition for the metric determined by $a_{k}, b_{k}$. Hence we have proved the first part of the following

Lemma 5.1. ( $i$ ) The zeros of the vector field given by (5.1a) and (5.1b) with $Y_{k} \neq 0$ for each $k$ correspond to Einstein metrics $g^{ \pm}$on the bundle $\bar{P}$.

(ii) Corresponding to these Einstein metrics we have $X_{k}=\frac{1}{m}$ and $Y_{k}=\frac{m}{\sqrt{d}} \beta^{ \pm}$where

$$
\beta^{ \pm}=\frac{(d+2)\left(1 \pm \sqrt{1-2\left(1+2 m^{-1}\right) d(d+2)^{-2}-3\left(1-m^{-1}\right)\left(1+2 m^{-1}\right)(d+2)^{-2}}\right)}{2 \sqrt{d}\left(2+3\left(1-m^{-1}\right) d^{-1}\right)} .
$$

Note that $\beta^{ \pm}$are the solutions of quadratic equation

$$
\left(2+\frac{3(m-1)}{m d}\right) \beta^{2}-\frac{d+2}{\sqrt{d}} \beta+\frac{m+2}{4 m}=0 .
$$

Proof. (ii) Since $E(X, Y)=\frac{m}{2}+1+4 m\left(\beta^{ \pm}\right)^{2}$, the (X,Y)'s in (ii) satisfy (5.2a) and (5.2b). It is a simple check that the discriminant of the quadratic equation is positive.

Remark 5.2. We shall refer to the above zeros as the Einstein points $\left(X_{E}^{ \pm}, Y_{E}^{ \pm}\right)$, where the \pm signs correspond to those occurring in $\beta^{ \pm}$. Note also that in Wa92 only one Einstein metric was found when $m \geq 3$.

The following estimates are needed for the discussion of eigenvalues below. 
Lemma 5.3. $\beta^{ \pm}$has the following lower and upper bounds:

$$
\begin{aligned}
& \frac{\sqrt{d}(d+2)}{2(2 d+3)}<\beta^{+}<\frac{\sqrt{d}(d+2)}{2(d+1)}, \\
& \frac{\sqrt{d}}{4(d+2)}<\beta^{-}<\frac{5 \sqrt{d}}{6(d+2)} .
\end{aligned}
$$

Proof. For the lower bound of $\beta^{+}$we compute

$$
\beta^{+}>\frac{d+2}{2 \sqrt{d}\left(2+3\left(1-m^{-1}\right) d^{-1}\right)}>\frac{d+2}{2 \sqrt{d}\left(2+3 d^{-1}\right)} .
$$

For the upper bound of $\beta^{+}$we compute

$$
\beta^{+}<\frac{2(d+2)}{2 \sqrt{d}\left(2+3\left(1-m^{-1}\right) d^{-1}\right)} \leq \frac{2(d+2)}{2 \sqrt{d}\left(2+2 d^{-1}\right)},
$$

where we used $m \geq 3$ to get the last inequality. This proves (5.3).

For the lower bound of $\beta^{-}$we compute using $\sqrt{1-\alpha} \leq 1-\frac{1}{2} \alpha$ for $\alpha \in[0,1]$

$$
\begin{aligned}
\beta^{-} & >\frac{(d+2)\left(\left(1+2 m^{-1}\right) d(d+2)^{-2}+\frac{3}{2}\left(1-m^{-1}\right)\left(1+2 m^{-1}\right)(d+2)^{-2}\right)}{2 \sqrt{d}\left(2+3\left(1-m^{-1}\right) d^{-1}\right)} \\
& >\frac{(d+2)^{-1}\left(\left(1+2 m^{-1}\right) d+\frac{3}{2}\right)}{2 \sqrt{d}\left(2+3\left(1-m^{-1}\right) d^{-1}\right)} \\
& >\frac{\sqrt{d}}{4(d+2)},
\end{aligned}
$$

where we have used $m \geq 3$ to get the last inequality. For the upper bound of $\beta^{-}$we compute using $\sqrt{1-\alpha} \geq 1-\alpha$ for $\alpha \in[0,1]$

$$
\begin{aligned}
\beta^{-} & <\frac{(d+2)^{-1}\left(2\left(1+2 m^{-1}\right) d+3\left(1-m^{-1}\right)\left(1+2 m^{-1}\right)\right)}{2 \sqrt{d}\left(2+3\left(1-m^{-1}\right) d^{-1}\right)} \\
& =\frac{(d+2)^{-1}}{2 \sqrt{d}} \cdot\left(1+2 m^{-1}\right) d .
\end{aligned}
$$

The lemma is proved.

Let $I_{m \times m}$ be the identity matrix and let $J$ be the $m \times m$ matrix all of whose entries are 1. Define $\mathcal{L}^{ \pm}$to be the linearization of the vector field from system (5.1a) and $(5.1 \mathrm{~b})$ at the Einstein points $\left(X_{E}^{ \pm}, Y_{E}^{ \pm}\right)$. By a straightforward computation we have

$$
\mathcal{L}^{ \pm}=\left(\begin{array}{cc}
c_{11}^{ \pm} I-8\left(\beta^{ \pm}\right)^{2} J & c_{12}^{ \pm} I-\frac{8 \sqrt{d}}{m^{2}} \beta^{ \pm} J \\
c_{21}^{ \pm} I+\frac{8 m^{2}}{\sqrt{d}}\left(\beta^{ \pm}\right)^{3} J & c_{22}^{ \pm} I+8\left(\beta^{ \pm}\right)^{2} J
\end{array}\right)_{2 m \times 2 m}
$$

where

$$
\begin{array}{ll}
c_{11}^{ \pm}=4(2 m-1)\left(\beta^{ \pm}\right)^{2}-\frac{m}{2}, & c_{12}^{ \pm}=\frac{8 \sqrt{d}}{m} \beta^{ \pm}>0, \\
c_{21}^{ \pm}=\frac{6 m^{2}(m-2)}{(\sqrt{d})^{3}}\left(\beta^{ \pm}\right)^{3}>0, & c_{22}^{ \pm}=\frac{12(m-1)}{d}\left(\beta^{ \pm}\right)^{2}-\frac{2(d+2) m}{\sqrt{d}} \beta^{ \pm} .
\end{array}
$$

Note that by the equation for $\beta^{ \pm}$we can rewrite $c_{22}^{ \pm}=\left(\frac{6(m-1)}{d}-4 m\right)\left(\beta^{ \pm}\right)^{2}-\frac{m+2}{2}$. 
We define two matrices

$$
C^{ \pm} \doteqdot\left(\begin{array}{cc}
c_{11}^{ \pm} & c_{12}^{ \pm} \\
c_{21}^{ \pm} & c_{22}^{ \pm}
\end{array}\right)
$$

Lemma 5.4. The eigenvalues of $C^{ \pm}$are real and distinct. If we denote by $\lambda_{1}^{ \pm}$and $\lambda_{2}^{ \pm}$the two eigenvalues of $C^{ \pm}$in ascending order, then

(i) $\lambda_{1}^{+}<0$ and $\lambda_{2}^{+}>0$, and

(ii) $\lambda_{1}^{-}<\lambda_{2}^{-}<0$.

Proof. The simplest way to deduce the first statement is to observe that $C^{ \pm}$can be written as the sum of a positive matrix and the scalar matrix $-\frac{2(d+2)}{\sqrt{d}} m \beta^{ \pm} I_{2 \times 2}$. The conclusion then follows from the Perron-Frobenius theorem for positive matrices.

(i) It suffices to prove that $c_{11}^{+}>0$ and $c_{22}^{+}<0$ which then imply that the determinant $\operatorname{det} C^{+}<0$. We consider two subcases.

Subcase (i1) $d \geq 2$. Using (5.3) we have

$$
\begin{aligned}
& c_{11}^{+}>4(2 m-1) \frac{d(d+2)^{2}}{4(2 d+4)^{2}}-\frac{m}{2}=\frac{1}{4}(2 m d-d-2 m)>0, \\
& c_{22}^{+}<\frac{2(d+2) \beta^{+}}{\sqrt{d}}\left(\frac{3(m-1)}{d+1}-m\right)<0 .
\end{aligned}
$$

Subcase (i2) $d=1$. By Lemma 5.1 we have $\beta^{+}=\frac{3 m+\sqrt{4 m^{2}-7 m+6}}{2(5 m-3)}$. Hence

$$
\frac{5 m-2}{2(5 m-3)}<\beta^{+}<\frac{5 m-1}{2(5 m-3)}
$$

Using these inequalities we can check that $c_{11}^{+}>0$ and $c_{22}^{+}<0$ when $d=1$.

(ii) First we prove trace $\operatorname{tr} C^{-}<0$ by considering two subcases.

Subcase (ii1) $d \geq 2$. We compute using (5.4)

$$
\begin{aligned}
\operatorname{tr} C^{-} & =\left(\frac{6(m-1)}{d}+4 m-4\right)\left(\beta^{-}\right)^{2}-(m+1) \\
& <\left(\frac{6(m-1)}{d}+4 m-4\right) \cdot \frac{25 d}{36(d+2)^{2}}-(m+1)
\end{aligned}
$$

The last expression as a function of $d \in[2, \infty)$ achieves its maximum when $d=2$, and the maximum value is negative. This proves $\operatorname{tr} C^{-}<0$ for $d \geq 2$.

Subcase (ii2) $d=1$. By Lemma 5.1 we have

$$
\frac{m+1}{2(5 m-3)}<\beta^{-}<\frac{m+2}{2(5 m-3)}
$$

From this we can calculate that $c_{11}^{-}=4(2 m-1)\left(\beta^{-}\right)^{2}-\frac{m}{2}<0$ and $c_{22}^{-}=(2 m-$ 6) $\left(\beta^{-}\right)^{2}-\frac{m+2}{2}<0$. Hence $\operatorname{tr} C^{-}<0$ when $d=1$. 
Next we show that $\operatorname{det} C^{-}>0$ for $m \geq 3$ and any $d$. We compute

$$
\begin{aligned}
\operatorname{det} C^{-}= & \frac{2 m \beta^{-}}{d}\left|\begin{array}{cc}
4(2 m-1)\left(\beta^{-}\right)^{2}-\frac{m}{2} & \frac{8 \sqrt{d}}{m} \beta^{-} \\
\frac{3 m(m-2)}{\sqrt{d}}\left(\beta^{-}\right)^{2} & \frac{6(m-1)}{m} \beta^{-}-(d+2) \sqrt{d}
\end{array}\right| \\
= & \frac{2 m \beta^{-}}{d}\left(\begin{array}{c}
24\left(m-1+m^{-1}\right)\left(\beta^{-}\right)^{3}-4 \sqrt{d}(d+2)(2 m-1)\left(\beta^{-}\right)^{2} \\
-3(m-1) \beta^{-}+\frac{1}{2} \sqrt{d}(d+2) m
\end{array}\right) \\
= & \frac{2 m \beta^{-}}{d}\left(\begin{array}{c}
-\left(8(2 m-1) d-12-12 m^{-1}\right)\left(\beta^{-}\right)^{3} \\
-\left(3(m-1)+\left(2 m+3-2 m^{-1}\right) d\right) \beta^{-}+\frac{1}{2} \sqrt{d}(d+2) m
\end{array}\right)
\end{aligned}
$$

where we have used the quadratic equation of $\beta^{-}$to get the last inequality. We define

$$
\Psi(m, d) \doteqdot\left(8(2 m-1) d-12-12 m^{-1}\right)\left(\beta^{-}\right)^{2}+3(m-1)+\left(2 m+3-2 m^{-1}\right) d .
$$

On the other hand when $m \geq 3$ and $d \geq 5$ we have

$$
\left(3 d^{2}+2 d-\frac{527}{9}\right) m>15 d
$$

which implies

$$
\left(\frac{127}{9} m+(2 m+3) d\right) \cdot \frac{5 \sqrt{d}}{6(d+2)}<\frac{1}{2} \sqrt{d}(d+2) m
$$

By the upper bound of $\beta^{-}$we get

$$
\left(\frac{127}{9} m+(2 m+3) d\right) \beta^{-}<\frac{1}{2} \sqrt{d}(d+2) m .
$$

Combining this with the fact that for $d \geq 2$ and $m \geq 3$, we have

$$
\begin{aligned}
\Psi(m, d) & <16 m d \cdot \frac{25 d}{36(d+2)^{2}}+3 m+(2 m+3) d \\
& <\frac{127}{9} m+(2 m+3) d
\end{aligned}
$$

and so we deduce that $-\Psi(m, d) \beta^{-}+\frac{1}{2} \sqrt{d}(d+2) m>0$. Hence $\operatorname{det} C^{-}>0$ when $m \geq 3$ and $d \geq 5$.

We have also shown that $\operatorname{det} C^{-}>0$ for $m \geq 3$ and $d=1,2,3,4$. This is done by using the specific value for $d$ and analysing the resulting expression for $\operatorname{det} C^{-}$. The calculations are straightforward but lengthy and not very illuminating so we omit them.

From $\operatorname{tr} C^{-}<0$ and $\operatorname{det} C^{-}>0$ we conclude that the eigenvalues of $C^{-}$are both negative.

Note that $\mathcal{L}^{ \pm}$maps linear subspace $V_{1} \doteqdot\left\{(X, Y) \in \mathbb{R}^{2 m}, \sum_{k=1}^{m} X_{k}=\sum_{k=1}^{m} Y_{k}=0\right\}$ into itself. Using the fact that $c_{12}^{ \pm}-\frac{8 \sqrt{d}}{m} \beta^{ \pm}=0$ it is easy to check that $\mathcal{L}^{ \pm}$in addition maps the linear subspace $V_{2} \doteqdot\left\{(X, Y) \in \mathbb{R}^{2 m}, \sum_{k=1}^{m} X_{k}=0\right\}$ into itself. This last property also follows from the fact that the affine hypersurface $\sum_{k=1}^{m} X_{k}=1$ is preserved by (5.1a). For the remainder of this section, we will regard $\mathcal{L}^{ \pm}$as a linear endomorphism of $V_{2}$ without introducing a separate notation for its restriction to $V_{2}$. 
Lemma 5.5. ( $i$ ) The matrices $\mathcal{L}^{ \pm}$are diagonalizable. They have distinct eigenvalues $\lambda_{1}^{ \pm}$and $\lambda_{2}^{ \pm}$and the corresponding eigenvectors form a basis of the linear subspace $V_{1}$. $\mathcal{L}^{ \pm}$have a further eigenvalue $\lambda_{3}^{ \pm} \doteqdot \frac{2(d+2) m}{\sqrt{d}} \beta^{ \pm}-(m+2)$ with associated eigenvector lying in $V_{2}$ but not in $V_{1}$. Furthermore, $\lambda_{3}^{+}>0$ while $\lambda_{3}^{-}<0$.

(ii) $\mathcal{L}^{+}$has $m$ positive eigenvalues and $m-1$ negative eigenvalues on $V_{2}$.

(iii) $\mathcal{L}^{-}$is negative definite on $V_{2}$.

Proof. (i) Let $\vec{u}=\left(u_{1}, \cdots, u_{m}\right)^{T}$ be any column vector which satisfies $\sum_{i=1}^{m} u_{i}=0$. Then we have

$$
\mathcal{L}^{ \pm}\left(\begin{array}{c}
\sigma_{1} \vec{u} \\
\sigma_{2} \vec{u}
\end{array}\right)=\left(\begin{array}{c}
\left(c_{11}^{ \pm} \sigma_{1}+c_{12}^{ \pm} \sigma_{2}\right) \vec{u} \\
\left(c_{21}^{ \pm} \sigma_{1}+c_{22}^{ \pm} \sigma_{2}\right) \vec{u}
\end{array}\right)=\lambda\left(\begin{array}{c}
\sigma_{1} \vec{u} \\
\sigma_{2} \vec{u}
\end{array}\right)
$$

provided that the column vector $\left(\sigma_{1}, \sigma_{2}\right)^{T} \in \mathbb{R}^{2}$ is an eigenvector of matrix $C^{ \pm}$with eigenvalue $\lambda$ :

$$
C^{ \pm}\left(\begin{array}{l}
\sigma_{1} \\
\sigma_{2}
\end{array}\right)=\lambda\left(\begin{array}{l}
\sigma_{1} \\
\sigma_{2}
\end{array}\right)
$$

Hence by Lemma 5.4 we obtain distinct eigenvalues $\lambda_{1}^{ \pm}$and $\lambda_{2}^{ \pm}$of $\mathcal{L}^{ \pm}$and the associated eigenvectors form a basis of the linear subspace $V_{1}$.

Next, let $\vec{e}$ be the column vector $(1, \cdots, 1)^{T}$. We compute that

$$
\mathcal{L}^{ \pm}\left(\begin{array}{c}
\sigma_{1} \vec{e} \\
\sigma_{2} \vec{e}
\end{array}\right)=\left(\begin{array}{c}
\left(c_{11}^{ \pm} \sigma_{1}-8 m\left(\beta^{ \pm}\right)^{2} \sigma_{1}+c_{12}^{ \pm} \sigma_{2}-\frac{8 \sqrt{d}}{m} \beta^{ \pm} \sigma_{2}\right) \vec{e} \\
\left(c_{21}^{ \pm} \sigma_{1}+\frac{8 m^{3}}{\sqrt{d}}\left(\beta^{ \pm}\right)^{3} \sigma_{1}+c_{22}^{ \pm} \sigma_{2}+8 m\left(\beta^{ \pm}\right)^{2} \sigma_{2}\right) \vec{e}
\end{array}\right)=\lambda\left(\begin{array}{c}
\sigma_{1} \vec{e} \\
\sigma_{2} \vec{e}
\end{array}\right)
$$

provided that the column vector $\left(\sigma_{1}, \sigma_{2}\right)^{T} \in \mathbb{R}^{2}$ is an eigenvector with eigenvalue $\lambda$ of matrix $D^{ \pm}$:

$$
D^{ \pm}\left(\begin{array}{c}
\sigma_{1} \\
\sigma_{2}
\end{array}\right) \doteqdot\left(\begin{array}{cc}
c_{11}^{ \pm}-8 m\left(\beta^{ \pm}\right)^{2} & c_{12}^{ \pm}-\frac{8 \sqrt{d}}{m} \beta^{ \pm} \\
c_{21}^{ \pm}+\frac{8 m^{3}}{\sqrt{d}}\left(\beta^{ \pm}\right)^{3} & c_{22}^{ \pm}+8 m\left(\beta^{ \pm}\right)^{2}
\end{array}\right)\left(\begin{array}{c}
\sigma_{1} \\
\sigma_{2}
\end{array}\right)=\lambda\left(\begin{array}{c}
\sigma_{1} \\
\sigma_{2}
\end{array}\right)
$$

Since

$$
D^{ \pm}=\left(\begin{array}{cc}
-4\left(\beta^{ \pm}\right)^{2}-\frac{m}{2} & 0 \\
\frac{6 m^{2}(m-2)+8 m^{3} d}{(\sqrt{d})^{3}}\left(\beta^{ \pm}\right)^{3} & \frac{8 m d+12(m-1)}{d}\left(\beta^{ \pm}\right)^{2}-\frac{2(d+2) m}{\sqrt{d}} \beta^{ \pm}
\end{array}\right),
$$

one sees that the vector $\left(\sigma_{1}, \sigma_{2}\right)^{T}=(0,1)^{T}$ is an eigenvector with eigenvalue

$$
\lambda_{3}^{ \pm}=\frac{8 m d+12(m-1)}{d}\left(\beta^{ \pm}\right)^{2}-\frac{2(d+2) m}{\sqrt{d}} \beta^{ \pm}=\frac{2(d+2) m}{\sqrt{d}} \beta^{ \pm}-(m+2) .
$$

This gives an eigenvector in the subspace $V_{2}$ which does not lie in $V_{1}$.

Note that using (5.3) we get

$$
\lambda_{3}^{+}>\frac{(d+2)^{2}}{2 d+3} m-(m+2)>0,
$$

while using (5.7) and (5.5) yields $\lambda_{3}^{-}<0$.

(ii) and (iii) These follow immediately from (i). 
5.2. Ancient solutions on $\bar{P}$ with $m \geq 3$. Now we can prove the existence of ancient solutions on the special class of fibre bundles $\bar{P}$ with $m \geq 3$ which were described at the beginning of $\S 5$.

Theorem 5.6. Let $\left(M_{i}^{4 n_{i}}, g_{i}\right), i=1, \cdots, m$, be $Q K$ manifolds with $\operatorname{Rc}_{g_{i}}=\Lambda_{i} g_{i}$. Assume $m \geq 3, n_{1}=\cdots=n_{m}=d$, and $\Lambda_{i}=d+2$ for all $i$. Let $g^{+}$and $g^{-}$be the Einstein metrics given in Lemma 5.1.

( $i)$ There is a continuous $(m-2)$-parameter family of ancient solutions of the Ricci flow on fibre bundle $\bar{P}$ such that $\lim _{\tau \rightarrow \infty} \frac{1}{\tau} \bar{g}(\tau)$ is a multiple of the Einstein metric $g^{+}$.

(ii) There is a continuous $(2 m-2)$-parameter family of ancient solution of the Ricci flow such that $\lim _{\tau \rightarrow \infty} \frac{1}{\tau} \bar{g}(\tau)$ is a multiple of the Einstein metric $g^{-}$.

(iii) All these ancient solutions are $\kappa$-noncollapsing, of type $I$ as $\tau \rightarrow \infty$, and have positive Ricci curvature when $\tau$ is large enough.

Proof. (i) We consider solutions $(X, Y)$ of the ODE system (5.1a) and (5.1b) in the space $\left\{(X, Y) \in \mathbb{R}^{2 m}: \sum_{k=1}^{m} X_{k}=1\right\}$. By Lemma 5.5 the linearized operator $\mathcal{L}^{+}$ at the Einstein point $\left(X_{E}^{+}, Y_{E}^{+}\right)$has a negative eigenvalue of multiplicity $m-1$ in $V_{2}$. So we may apply the Hartman-Grobman Theorem to conclude that there is a continuous $(m-2)$-parameter family of solutions $\left(X^{+}(u), Y^{+}(u)\right)$ whose limits $\lim _{u \rightarrow \infty}\left(X^{+}(u), Y^{+}(u)\right)=\left(X_{E}^{+}, Y_{E}^{+}\right)$. By the discussion after equation (5.1b) we get a continuous $(m-2)$-parameter family of solutions of the backwards Ricci flow (modulo time translation and parabolic scaling). To see that these solutions are ancient, we prove next that $\lim _{u \rightarrow \infty} \tau(u) \rightarrow \infty$.

If we take a flow line $\left(X^{+}(u), Y^{+}(u)\right)$ in the local stable manifold near $\left(X_{E}^{+}, Y_{E}^{+}\right)$, then $\lim _{u \rightarrow \infty}\left(X^{+}(u), Y^{+}(u)\right)=\left(X_{E}^{+}, Y_{E}^{+}\right)$and $\lim _{u \rightarrow \infty} E\left(X^{+}(u), Y^{+}(u)\right)=E\left(X_{E}^{+}, Y_{E}^{+}\right)$. Hence given any $\varepsilon>0$ there is a $u_{0}$ such that for $u \geq u_{0}$ we have

$$
\left|E\left(X^{+}(u), Y^{+}(u)\right)-E\left(X_{E}^{+}, Y_{E}^{+}\right)\right| \leq \varepsilon .
$$

We compute by using $\frac{d}{d u} \ln \hat{a}(u)=E\left(X^{+}(u), Y^{+}(u)\right)$ that

$$
\ln \hat{a}(u)-\ln \hat{a}\left(u_{0}\right) \leq\left(E\left(X_{E}^{+}, Y_{E}^{+}\right)+\varepsilon\right)\left(u-u_{0}\right) \quad \text { for } u \geq u_{0} .
$$

Hence we have

$$
\hat{a}(u) \leq \hat{a}\left(u_{0}\right) e^{\left(E\left(X_{E}^{+}, Y_{E}^{+}\right)+\varepsilon\right)\left(u-u_{0}\right)} \quad \text { for } u \geq u_{0} .
$$

This and the relation $d \tau=\hat{a}(u) d u$ imply that $\lim _{u \rightarrow \infty} \tau(u) \rightarrow \infty$.

From (2.4) and (2.1) we get

$$
\begin{aligned}
& \frac{d a_{k}}{d \tau}=\frac{1}{2}+X_{k}+4 d X_{k}^{2} Y_{k}^{2} \rightarrow \frac{m+2}{2 m}+4\left(\beta^{+}\right)^{2} \\
& \frac{d b_{k}}{d \tau}=2(d+2)-6 X_{k}\left(1-X_{k}\right) Y_{k} \rightarrow 2(d+2)-\frac{6(m-1)}{m \sqrt{d}} \beta^{+}>0
\end{aligned}
$$

as $\tau \rightarrow \infty$. We have used (5.3) to get the last inequality. This implies that $\lim _{\tau \rightarrow \infty} \frac{a_{k}}{\tau}$ and $\lim _{\tau \rightarrow \infty} \frac{b_{k}}{\tau}$ exist. (i) is now proved.

(ii) Note that the linearized operator $\mathcal{L}^{-}$at the Einstein point $\left(X_{E}^{-}, Y_{E}^{-}\right)$has $2 m-1$ negative eigenvalues in $V_{2}$ by Lemma 5.5. With this observation, the rest of the proof is similar to the proof of (i), and so we omit it. 
(iii) The $\kappa$-noncollapsing and Type I properties follow from the fact that $\lim _{\tau \rightarrow \infty} \frac{\bar{g}(\tau)}{\tau}$ is a multiple of the nonflat Einstein metrics $g^{ \pm}$and Corollary 4.4.

From [Wa92, (2.2)], the restriction of the Ricci tensor of $\bar{g}(\tau)$ to the fibre directions is positive definite. So by (2.1) ) the Ricci tensor is positive definite if and only if

$$
2(d+2)-6 X_{k}\left(1-X_{k}\right) Y_{k}>0, \quad \text { for } k=1, \cdots, m .
$$

These inequalities hold when $(X(\tau), Y(\tau))$ are close to $\left(X_{E}^{ \pm}, Y_{E}^{ \pm}\right)$.

Remark 5.7. (i) The dimensions of the bundles considered in this section are of the form $4 m d+3(m-1)$. In particular, for $m=2 l+1$ with $l \geq 1$, the dimension is even. However, the total spaces of the bundles cannot admit a Kähler structure if none of the QK factors in the base are complex Grassmannian. To see this, recall that S. Salamon [Be87, 14.83] has proved that positive QK manifolds are simply connected, and that the rigidity result of LeBrun and Salamon [LS94] implies that under our assumption the second Betti number of the base is 0. As well, the fibres are products of $\mathbb{R P}^{3}$, which is a homology 3 -sphere. It then follows for example from the Serre spectral sequence that the second Betti number of the total space is trivial. So the metrics in our ancient flows do not admit compatible symplectic structures which are non-cohomologous to zero. The only other ancient solutions of this type that we know of are the continuous families on compact simple Lie groups constructed by Lauret [La13] and the isolated solutions on the homogeneous spaces $\operatorname{Sp}(3) /(\operatorname{Sp}(1) \times \operatorname{Sp}(1) \times \operatorname{Sp}(1))$ and $F_{4} / \operatorname{Spin}(8)$ (see Example 5 in [BKN12]).

(ii) We also obtain the pseudo-Riemannian Ricci flow analogs of the ancient solutions in Theorem 5.6 by the same arguments in Remark 3.2 .

\section{REFERENCES}

[BKN12] I. Bakas, S. Kong, and L. Ni, Ancient solutions of Ricci flow on spheres and generalized Hopf fibrations. J. Reine Angew. Math., 663 (2012), 209-248.

[Be87] A. Besse, Einstein manifolds. Springer-Verlag, Berlin, 1987.

[CGT82] J. Cheeger, M. Gromov, and M. Taylor, Finite propagation speed, kernel estimates for functions of the Laplace operator, and the geometry of complete Riemannian manifolds. J. Diff. Geom., 17 (1982), 15-53.

[Co96] V. Cortés, Alekseevskian spaces. Diff. Geom. Appl., 6 (1996), 129-168.

[DHS12] P. Daskalopoulos, R. Hamilton, and N. Sesum, Classification of compact ancient solutions to the Ricci flow on surfaces. J. Diff. Geom., 91 (2012), 171-214.

[Dy15] M. Dyckmanns, The hyper-Kähler and quaternionic Kähler correspondence and the geometry of the c-map. Dissertation Hamburg University, 2015.

[Ga59] F.R. Gantmacher, Applications of the theory of matrices. Interscience Publishers, Inc., New York, 1959.

[Ha93] R. Hamilton, The formation of singularities in the Ricci flow. Surveys in differential geometry, Vol. II (Cambridge, MA, 1993), 7-136, Internat. Press, Cambridge, MA, 1995.

[Hi81] N. Hitchin, Kählerian twistor spaces. Proc. LMS., 43 (1981), 133-150.

[La13] J. Lauret, Ricci flow of homogeneous manifolds. Math. Z., 274 (2013), 373-403.

[Le91] C. Lebrun, On complete quaternionic-Kähler manifolds. Duke Math. J., 63 (1991), 723-743.

[LS94] C. Lebrun and S. Salamon, Strong rigidity of positive quaternionic-Kähler manifolds. Invent. Math., 118 (1994) 109-132.

[LW16] P. Lu and Y.K. Wang, Ancient Ricci flow solutions on bundles. arXiv: 1606.01112.

[MS88] M. Mamone and S. Salamon, Yang-Mills fileds on quanternionic spaces. Nonlinearity, 1 (1988), 517-530. 
[Pe02] G. Perelman, The entropy formula for the Ricci flow and its geometric applications. arXiv:math.DG/0211159.

[PS91] Y. S. Poon and S. Salamon, Quaternionic Kähler 8-manifolds with positive scalar curvature. J. Diff. Geom., 33 (1991), 363-378.

[Sa89] S. Salamon, Riemannian geometry and holonomy groups. Longman Scientific and Technical, 1989.

[St57] S. Sternberg, Local contractions and a theorem of Poincaré. Amer. J. Math., 79 (1957), 809-824.

[Wa92] M. Wang, Einstein metrics and quaternionic Kähler manifolds. Math. Z., 210 (1992), 305325 .

Department of Mathematics, University of Oregon, Eugene, OR 97403-1222

E-mail address: penglu@uoregon.edu

Department of Mathematics and Statistics, McMaster University, Hamilton, OnTARIO, L8S 4K1, CANADA

E-mail address: wang@mcmaster.ca 